\title{
Exactly solvable quantum state reduction models with time-dependent coupling
}

\author{
Dorje C. Brody*, Irene C. Constantinou*, James D. C. Dear ${ }^{\dagger}$, and Lane P. Hughston ${ }^{\dagger}$ \\ *Blackett Laboratory, Imperial College, London SW7 2BZ, UK \\ †Department of Mathematics, King's College London, \\ The Strand, London WC2R 2LS, UK
}

(Dated: August 20, 2018)

\begin{abstract}
A closed-form solution to the energy-based stochastic Schrödinger equation with a time-dependent coupling is obtained. The solution is algebraic in character, and is expressed directly in terms of independent random data. The data consist of (i) a random variable $H$ which has the distribution $\mathbb{P}\left(H=E_{i}\right)=\pi_{i}$, where $\pi_{i}$ is the transition probability $\left|\left\langle\psi_{0} \mid \phi_{i}\right\rangle\right|^{2}$ from the initial state $\left|\psi_{0}\right\rangle$ to the Lüders state $\left|\phi_{i}\right\rangle$ with energy $E_{i}$; and (ii) an independent $\mathbb{P}$-Brownian motion, where $\mathbb{P}$ is the physical probability measure associated with the dynamics of the reduction process. When the coupling is time-independent, it is known that state reduction occurs asymptotically - that is to say, over an infinite time horizon. In the case of a time-dependent coupling, we show that if the magnitude of the coupling decreases sufficiently rapidly, then the energy variance will be reduced under the dynamics, but the state need not reach an energy eigenstate. This situation corresponds to the case of a "partial" or "incomplete" measurement of the energy. We also construct an example of a model where the opposite situation prevails, in which complete state reduction is achieved after the passage of a finite period of time.
\end{abstract}

PACS numbers: 03.65.Ta, 02.50.Ey, 02.50.Ga, 02,50,Cw

\section{INTRODUCTION}

This paper is concerned with the problem of obtaining closed-form solutions to the energybased stochastic extension of the Schrödinger equation in the case of a time-dependent coupling parameter. In this situation the dynamical equation of the wave function is assumed to satisfy the following stochastic differential equation:

$$
\mathrm{d}\left|\psi_{t}\right\rangle=-\mathrm{i} \hat{H}\left|\psi_{t}\right\rangle \mathrm{d} t-\frac{1}{8} \sigma_{t}^{2}\left(\hat{H}-H_{t}\right)^{2}\left|\psi_{t}\right\rangle \mathrm{d} t+\frac{1}{2} \sigma_{t}\left(\hat{H}-H_{t}\right)\left|\psi_{t}\right\rangle \mathrm{d} W_{t} .
$$

Here $\left\{\left|\psi_{t}\right\rangle\right\}_{0 \leq t<\infty}$ denotes the state-vector process, which is defined on a probability space $(\Omega, \mathcal{F}, \mathbb{P})$ with filtration $\left\{\mathcal{F}_{t}\right\}_{0 \leq t<\infty}$, with respect to which $\left\{W_{t}\right\}_{0 \leq t<\infty}$ is a standard onedimensional Brownian motion. The operator $\hat{H}$ is the Hamiltonian of the system, and

$$
H_{t}=\left\langle\psi_{t}|\hat{H}| \psi_{t}\right\rangle
$$

is the expectation value of $\hat{H}$ in the state $\left|\psi_{t}\right\rangle$. The time-dependent coupling parameter $\left\{\sigma_{t}\right\}_{0 \leq t<\infty}$, which has the units [energy $]^{-1}[\text { time }]^{-1 / 2}$, and is assumed to be a given positive function, determines the rate at which state-vector reduction occurs. For simplicity we consider the case of a pure initial state $\left|\psi_{0}\right\rangle$; the generalisation to the case of a mixed initial state is straightforward. For convenience we assume that the initial state has norm unity; a 
straightforward exercise making use of the Ito calculus then shows that $\left\langle\psi_{t} \mid \psi_{t}\right\rangle=1$ for all $t \geq 0$. Likewise for simplicity we assume that the Hamiltonian $\hat{H}$ has a discrete spectrum, and that the Hilbert space is of finite dimension. We assume that the possible values of the energy are given by $\left\{E_{i}\right\}_{i=1,2, \ldots, N}$. The transition probability from the initial state $\left|\psi_{0}\right\rangle$ to a state of energy $E_{i}$ is $\pi_{i}=\left\langle\psi_{0}\left|\hat{\Pi}_{i}\right| \psi_{0}\right\rangle$, where $\hat{\Pi}_{i}$ denotes the projection operator onto the Hilbert subspace of states with energy $E_{i}$. Equivalently, we can write $\pi_{i}=\left|\left\langle\phi_{i} \mid \psi_{0}\right\rangle\right|^{2}$, where $\left|\phi_{i}\right\rangle=\pi_{i}^{-1 / 2} \hat{\Pi}_{i}\left|\psi_{0}\right\rangle$ is the so-called Lüders state associated with the given initial state $\left|\psi_{0}\right\rangle$ and the energy $E_{i}$. We note that $\left\langle\phi_{i} \mid \phi_{i}\right\rangle=1$. According to the von Neumann-Lüders state-vector reduction hypothesis [4, 25, 30], if the initial state of the quantum system is $\left|\psi_{0}\right\rangle$ and a measurement of the energy is made with the result $E_{i}$, then there is a discontinuous transformation of the state of the system, and the new state is given by $\left|\phi_{i}\right\rangle$. In particular, in the case of a degenerate spectrum a specific state is selected in this way among all those with the given eigenvalue.

In the stochastic framework the discontinuous von Neumann-Lüders reduction process is replaced by a continuous reduction process modelled by equation (11). Depending on the details of the physical setup, the reduction process modelled by (11) can be regarded as taking place either (a) as a consequence of a measurement having been made, or (b) as a result of interaction of the system with its environment, or (c) spontaneously. For instance, we can view (11) as a phenomenological "reduced-form" model for the dynamics of a system when an energy measurement is made. The time-dependent coupling in that case represents the exogenous intervention of the measurement apparatus in the dynamics of the system.

The mathematical and physical properties of the energy-based stochastic extension of the Schrödinger equation have been studied extensively in the literature [1, 2, 3, 4, 6, 9, 10, 11, 12, 13, 22, 24, 32, 33]. The energy-based stochastic Schrödinger equation can be regarded as a special case of a more general class of stochastic models for the dynamics of the wave function that use nonlinear stochastic differential equations of the form (11), but typically involving a number of quantum operators. Such models have been introduced with a variety of aims, and in the general situation the operators need not commute amongst themselves or with the Hamiltonian, and need not be Hermitian. We mention, e.g., [5, 7, 8, 15, 19, 21, 23, 31, 34], and works cited therein, as references to the substantial body of publications in this area.

The significance of the energy-based reduction model, in contrast with the more general situation, is that energy is conserved in expectation. More specifically, it is a property of the dynamical equation (II) that $\operatorname{tr}\left(\hat{\rho}_{t} \hat{H}\right)$ is constant, where the time-dependent density matrix $\hat{\rho}_{t}$ is defined by $\hat{\rho}_{t}=\mathbb{E}^{\mathbb{P}}\left[\left|\psi_{t}\right\rangle\left\langle\psi_{t}\right|\right]$. Here $\mathbb{E}^{\mathbb{P}}[-]$ denotes expectation with respect to the physical probability measure $\mathbb{P}$. Conservation of the energy follows from the fact that

$$
\frac{\mathrm{d} \hat{\rho}_{t}}{\mathrm{~d} t}=-\mathrm{i}\left[\hat{H}, \hat{\rho}_{t}\right]+\frac{1}{4} \sigma_{t}^{2}\left[\hat{H} \hat{\rho}_{t} \hat{H}-\frac{1}{2} \hat{H}^{2} \hat{\rho}_{t}-\frac{1}{2} \hat{\rho}_{t} \hat{H}^{2}\right] .
$$

Thus, the energy-based stochastic Schrödinger equation can be regarded as appropriate to the description of the dynamics of an isolated system, or any system for which on average there is no net exchange of energy with the environment.

When the coupling parameter in equation (11) is a constant, it is possible to obtain a closed-form solution to this stochastic differential equation [10, 11, 12, 13]. The solution to (11) in the case of a constant coupling parameter can at each time $t$ be expressed as a function of a state variable $\xi_{t}$, the value of which is determined by the specification of a pair of independent random data corresponding roughly to the idea of a split between "signal" and "noise". More specifically, the state-variable process $\left\{\xi_{t}\right\}_{0 \leq t<\infty}$ is of the form $\xi_{t}=\sigma H t+B_{t}$, 
where $H$ is a random variable that takes the value $E_{i}(i=1,2, \ldots, N)$ with probability $\pi_{i}$, and $\left\{B_{t}\right\}_{0 \leq t<\infty}$ is an independent Brownian motion. The purpose of this paper is to show how this construction can be generalised to the case of a generic time-dependent coupling.

The paper is organised as follows. In Section 1 we introduce an ansatz that leads to the solution of (II). Some comments are made on the interpretation of the ansatz and its relation to similar constructions in the theory of filtering. In Section [1] we obtain general expressions for the conditional probability $\pi_{i t}=\mathbb{E}\left[\mathbf{1}_{\left\{H=E_{i}\right\}} \mid \mathcal{F}_{t}\right]$ and the conditional energy expectation $H_{t}=\mathbb{E}\left[H \mid \mathcal{F}_{t}\right]$ in terms of the process $\left\{\xi_{t}\right\}_{0 \leq t<\infty}$. Whereas in the time-independent case the random variable $H_{t}$ can be expressed, for each value of $t$, as a function of the state variable $\xi_{t}$, in the time-dependent case $H_{t}$ is a functional of the trajectory $\left\{\xi_{s}\right\}_{0 \leq s \leq t}$. In Section IV we show that although $\left\{\xi_{t}\right\}$ is in general non-Markovian, the corresponding energy process $\left\{H_{t}\right\}$, which is a functional of $\left\{\xi_{t}\right\}$, does have the Markov property.

In Section $\nabla$ we show how the analysis of Section III leads to an expression for the state-vector process that solves (II). In Section [V] we examine the corresponding "inverse" problem. We show how, given any random trajectory $\left|\psi_{t}\right\rangle$ that solves (1), it is possible to construct explicitly the independent random data $H$ and $\left\{B_{t}\right\}$ associated with it. We also remark on the relation between our solution technique and a well-known linearisation method often used in analysing the properties of (11). Then in Section VII] we show that the state vector collapses to the specified eigenstate, provided a condition on the coupling is satisfied.

More generally, since we consider an essentially arbitrary time-dependent coupling, one can envisage circumstances in which the coupling ceases before state reduction is complete. This kind of situation can be regarded as representing an approximate measurement of the energy, in which partial information is gained but no definite outcome is obtained. In Section VIII we examine this case in some detail, and derive upper and lower bounds for the asymptotic value of the energy variance as $t$ goes to infinity.

Another interesting situation arises when the magnitude of the coupling increases sufficiently rapidly to ensure that state reduction is completed after the passage of a finite amount of time. In Section [X] a special example of such a model is constructed. This example turns out to have a direct relationship to the finite-time collapse model introduced in 11]. The model presented in [11] is based on a constant coupling parameter and a Brownian bridge noise. Here we present a alternative model for finite-time collapse, for which the noise is a standard Brownian motion and the coupling is time-dependent. We demonstrate that the two models are physically equivalent.

While the probabilistic method presented in Section III is effective in obtaining the solution to the problem under consideration here, there are circumstances in which other methods are useful as well. We thus outline two further approaches for obtaining the solution. In Appendix $\$$ we introduce a method that involves a discretisation and a continuum limit, and proceeds in a manner similar to the analysis entailed in the evaluation of Feynman integrals. The method is computationally intense, but is satisfying because it allows one to work directly with the quantities under investigation. In Appendix B we consider another method that is similar to the path integral approach, except that we use a decomposition of the state-variable trajectory into increments, and we regard the time-dependent coupling as moderating the noise rather than the signal. 


\section{THE QUANTUM INFORMATION PROCESS}

The ansatz that we use to solve (11) is based on the specification of a state-variable process $\left\{\xi_{t}\right\}$ which, for reasons discussed shortly, will be called the "quantum information process", and is of the following form:

$$
\xi_{t}=H \int_{0}^{t} \sigma_{s} \mathrm{~d} s+B_{t} .
$$

Here $H$ denotes a random variable on the given probability space $(\Omega, \mathcal{F}, \mathbb{P})$, taking the possible values $\left\{E_{i}\right\}_{i=1,2, \ldots, N}$ with the probabilities $\left\{\pi_{i}\right\}_{i=1,2, \ldots, N}$, and $\left\{B_{t}\right\}_{0 \leq t<\infty}$ is a standard Brownian motion, independent of $H$. We do not assume that $\left\{B_{t}\right\}$ is adapted to the filtration $\left\{\mathcal{F}_{t}\right\}$ introduced earlier. On the contrary, we shall see later that $\left\{\mathcal{F}_{t}\right\}$ is generated by $\left\{\xi_{t}\right\}$. The various terms appearing in (4) can be given an interpretation in the language of filtering theory. This "signal and noise" interpretation, although not essential to the use of the ansatz to solve (11), is nonetheless physically very suggestive, and as a consequence helps to motivate the form that the solution takes. Indeed, the methodology of filtering theory has been already shown [10] to be effective in deriving solutions to the energy-based stochastic Schrödinger equation, and in what follows we take this line of investigation further.

The random variable $H$, according to this interpretation, represents the unknown terminal value of the energy of the quantum system whose time evolution is described by equation (11). The term $H \int_{0}^{t} \sigma_{s} \mathrm{~d} s$ in (44) should be thought of as the "signal" component of the quantum information process. As time passes, the magnitude of the signal component increases, but the true value of $H$ remains obscured by the presence of a noise process $\left\{B_{t}\right\}_{0 \leq t<\infty}$. The "accessible" information concerning the value of $H$ is represented by the process $\left\{\xi_{t}\right\}_{0 \leq t<\infty}$, which consists of both signal and noise. Given the history $\left\{\xi_{s}\right\}_{0 \leq s<t}$ over a finite time interval $[0, t]$, it is not generally possible to disentangle the true value of $H$ from the noise. In the context of filtering theory, the task in such a setup is to determine the best estimate of $H$, given the information of the trajectory $\left\{\xi_{s}\right\}_{0 \leq s \leq t}$ from time zero to time $t$. It is a remarkable feature of the stochastic Schrödinger equation that the expectation of the Hamiltonian turns out to be given by such an estimate.

In the first part of the paper we shall examine the case for which the state-vector trajectory $\left\{\left|\psi_{t}\right\rangle\right\}$ satisfies the dynamical equation (11) for all $t \in[0, \infty)$. In order for the trajectory $\left\{\left|\psi_{t}\right\rangle\right\}_{0 \leq t<\infty}$ to be well-defined, the coupling function $\left\{\sigma_{t}\right\}_{0 \leq t<\infty}$ must be such that

$$
\int_{0}^{t} \sigma_{s}^{2} \mathrm{~d} s<\infty, \quad 0 \leq t<\infty .
$$

Additionally, as will be established in Section VII to ensure a complete reduction of the state vector, the coupling function must be chosen such that

$$
\lim _{t \rightarrow \infty} \int_{0}^{t} \sigma_{s}^{2} \mathrm{~d} s=\infty .
$$

The purpose of this condition is to ensure that the coupling remains reasonably "strong" for all time, and does not attenuate too much.

There are circumstances in which (5) is satisfied but (6) is not. In such situations the wave function need not fully collapse to an eigenstate, even though the energy variance will be reduced. This case is examined in Section VIII. It will be assumed throughout Sections 
III VIII that $\left\{\xi_{t}\right\}$ and $\left\{\sigma_{t}\right\}$ are defined for all $t$ in the range $0 \leq t<\infty$, and that (5) holds. In Section $[\mathbb{X}]$ we drop the assumption of an infinite collapse time, and consider the case for which the integral of $\left\{\sigma_{t}\right\}$ diverges after a finite passage of time.

The estimation problem posed by an ansatz of the form (4) is well established in the literature of nonlinear filtering [14, 29]. The relevance of (41) to the dynamics of the quantum state $\left\{\left|\psi_{t}\right\rangle\right\}$ satisfying the stochastic Schrödinger equation (1D), on the other hand, is not obvious. As we shall demonstrate, the information generated by $\left\{\xi_{s}\right\}_{0 \leq s \leq t}$ is equivalent to the information generated by the evolution $\left\{\left|\psi_{s}\right\rangle\right\}_{0 \leq s \leq t}$ of the quantum state itself. We formalise this notion by observing that $\left\{\xi_{t}\right\}_{0 \leq t<\infty}$ and $\left\{\left|\psi_{t}\right\rangle\right\}_{0 \leq t<\infty}$ generate the same filtration $\left\{\mathcal{F}_{t}\right\}_{0 \leq t<\infty}$. As a consequence, the energy process $\left\{H_{t}\right\}$ determined by the quantum expectation (2) of the Hamiltonian operator turns out to be indistinguishable from the process generated as $t$ varies by the mathematical expectation of the random variable $H$, conditional on the specification of the trajectory $\left\{\xi_{s}\right\}_{0 \leq s \leq t}$.

The best estimate for $H$, in the sense of least quadratic error, given the history of the information process up to time $t$, is known (see, e.g., [10]) to be the conditional expectation

$$
H_{t}=\mathbb{E}\left[H \mid\left\{\xi_{s}\right\}_{0 \leq s \leq t}\right] .
$$

We have used the same notation $\left\{H_{t}\right\}$ for the processes defined in (2) and in (77) because these processes will be shown to be the same. When $\left\{\sigma_{t}\right\}$ is constant, equation (17) can be simplified to the form $H_{t}=\mathbb{E}\left[H \mid \xi_{t}\right]$. In this case, $\left\{\xi_{t}\right\}$ is Markovian: this is the situation considered in [10, 11, 12, 13]. However, if $\left\{\sigma_{t}\right\}$ is not constant, then in general the trajectory $\left\{\xi_{s}\right\}_{0 \leq s \leq t}$ must be taken into account to determine the conditional expectation (17). The nonMarkovian property of $\left\{\xi_{t}\right\}$ can be seen intuitively as follows. Writing (4) in differential form, we have

$$
\mathrm{d} \xi_{t}=\sigma_{t} H \mathrm{~d} t+\mathrm{d} B_{t}
$$

which makes it evident that $\left\{\sigma_{t}\right\}$ determines the strength of the signal, that is to say, the rate at which the true value of $H$ is revealed. If $\left\{\sigma_{t}\right\}$ is constant, then sampling from $\left\{\xi_{t}\right\}$ at any small time period in the interval $[0, t]$ is as good as any other. This is, in essence, the property of $\left\{\xi_{t}\right\}$, when $\left\{\sigma_{t}\right\}$ is constant, that makes it Markovian. If $\left\{\sigma_{t}\right\}$ is not constant, then there is a temporal bias in the sampling from $\left\{\xi_{t}\right\}$, and observations from different periods cannot be treated on an equal footing.

\section{CONDITIONAL PROBABILITY PROCESS}

Our goal in this section is to work out an explicit expression for the conditional probability process $\left\{\pi_{i t}\right\}$ defined by

$$
\pi_{i t}=\mathbb{E}\left[\mathbf{1}_{\left\{H=E_{i}\right\}} \mid\left\{\xi_{s}\right\}_{0 \leq s \leq t}\right] .
$$

Here $\mathbf{1}_{\{A\}}$ denotes the indicator function which takes the value unity if $A$ is true, and zero if $A$ is false. Once we obtain $\pi_{i t}$, then the conditional expectation (77) can be obtained by the relation $H_{t}=\sum_{i} \pi_{i t} E_{i}$.

To determine $\left\{\pi_{i t}\right\}$ we use a change-of-measure technique (see, e.g., [14, 17, 26] ), proceeding as follows. Let $(\Omega, \mathcal{F}, \mathbb{P})$ be a probability space on which a standard Brownian motion 
$\left\{B_{t}\right\}$ is defined, and let $H$ be a random variable on $(\Omega, \mathcal{F}, \mathbb{P})$ that is independent of $\left\{B_{t}\right\}$. We fix a time interval $[0, u]$, and let $\left\{\xi_{t}\right\}_{0 \leq t \leq u}$ be given by

$$
\xi_{t}=H \int_{0}^{t} \sigma_{s} \mathrm{~d} s+B_{t}
$$

where $\left\{\sigma_{t}\right\}$ is deterministic and satisfies (5). Next, we define a process $\left\{\Lambda_{t}\right\}_{0 \leq t \leq u}$ over $[0, u]$ by the expression

$$
\Lambda_{t}=\exp \left(H \int_{0}^{t} \sigma_{s} \mathrm{~d} \xi_{s}-\frac{1}{2} H^{2} \int_{0}^{t} \sigma_{s}^{2} \mathrm{~d} s\right),
$$

or equivalently, by virtue of (10),

$$
\Lambda_{t}^{-1}=\exp \left(-H \int_{0}^{t} \sigma_{s} \mathrm{~d} B_{s}-\frac{1}{2} H^{2} \int_{0}^{t} \sigma_{s}^{2} \mathrm{~d} s\right) .
$$

The idea is to use $\left\{\Lambda_{t}\right\}$ to make a change of probability measure. The new probability measure will be defined on the space $\left(\Omega, \mathcal{G}_{u}\right)$, where $\mathcal{G}_{u} \subset \mathcal{F}$ is the $\sigma$-subalgebra of events determined by the specification of the trajectory $\left\{B_{t}\right\}_{0 \leq t \leq u}$ over the given time horizon, together with $H$.

We recall that the points of $\Omega$ represent the possible outcomes of chance, and the elements of $\mathcal{F}$ are subsets of $\Omega$ with the property that for each such subset $A \in \mathcal{F}$ the measure $\mathbb{P}$ assigns a probability $\mathbb{P}(A)$ to the event that $\omega \in A$. The elements of $\mathcal{G}_{u}$ consist of those elements $A \in \mathcal{F}$ with the property that knowledge of the value of $H$ and the trajectory $\left\{B_{t}\right\}_{0 \leq t \leq u}$ is sufficient to determine whether $\omega \in A$. For any element $A \in \mathcal{G}_{u}$ the value of the indicator function $\mathbf{1}_{\{\omega \in A\}}$ is determined by the specification of $H$ and $\left\{B_{t}\right\}_{0 \leq t \leq u}$. The new measure $\mathbb{Q}$ on $\left(\Omega, \mathcal{G}_{u}\right)$ is then given as follows. For any set $A \in \mathcal{G}_{u}$ we define

$$
\mathbb{Q}(A)=\mathbb{E}^{\mathbb{P}}\left[\Lambda_{u}^{-1} \mathbf{1}_{\{\omega \in A\}}\right]
$$

This relation is usually abbreviated by writing $\mathrm{d} \mathbb{Q}=\Lambda_{u}^{-1} \mathrm{~d} \mathbb{P}$. Since $\mathbb{E}^{\mathbb{P}}\left[\Lambda_{u}^{-1}\right]=1$ by virtue of elementary properties of the stochastic exponential (12), it follows that $\mathbb{Q}(\Omega)=1$.

Given the setup described above, we have the following facts: (i) on the probability space $\left(\Omega, \mathcal{G}_{u}, \mathbb{Q}\right)$, the process $\left\{\xi_{t}\right\}_{0 \leq t \leq u}$ defined by (10) is a Brownian motion, and is independent of $H$; (ii) the random variable $H$ has the same probability law with respect to $\mathbb{Q}$ as it does with respect to $\mathbb{P}$; (iii) for all $t \in[0, u]$, the conditional expectation $f_{t}=\mathbb{E}^{\mathbb{P}}\left[f(H) \mid\left\{\xi_{s}\right\}_{0 \leq s \leq t}\right]$ of a function of the random variable $H$ can be expressed in the form

$$
f_{t}=\frac{\mathbb{E}^{\mathbb{Q}}\left[f(H) \Lambda_{t} \mid\left\{\xi_{s}\right\}_{0 \leq s \leq t}\right]}{\mathbb{E}^{\mathbb{Q}}\left[\Lambda_{t} \mid\left\{\xi_{s}\right\}_{0 \leq s \leq t}\right]} .
$$

To work out an expression for $\pi_{i t}$ we start with the definition (91), substitute (11) into (14) and set $f(H)=\mathbf{1}_{\left\{H=E_{i}\right\}}$ to obtain

$$
\pi_{i t}=\frac{\pi_{i} \exp \left(E_{i} \int_{0}^{t} \sigma_{s} \mathrm{~d} \xi_{s}-\frac{1}{2} E_{i}^{2} \int_{0}^{t} \sigma_{s}^{2} \mathrm{~d} s\right)}{\sum_{i} \pi_{i} \exp \left(E_{i} \int_{0}^{t} \sigma_{s} \mathrm{~d} \xi_{s}-\frac{1}{2} E_{i}^{2} \int_{0}^{t} \sigma_{s}^{2} \mathrm{~d} s\right)} .
$$


Similarly, by setting $f(H)=H$ we obtain

$$
H_{t}=\frac{\sum_{i} \pi_{i} E_{i} \exp \left(E_{i} \int_{0}^{t} \sigma_{s} \mathrm{~d} \xi_{s}-\frac{1}{2} E_{i}^{2} \int_{0}^{t} \sigma_{s}^{2} \mathrm{~d} s\right)}{\sum_{i} \pi_{i} \exp \left(E_{i} \int_{0}^{t} \sigma_{s} \mathrm{~d} \xi_{s}-\frac{1}{2} E_{i}^{2} \int_{0}^{t} \sigma_{s}^{2} \mathrm{~d} s\right)} .
$$

The result appears at first glance to depend on the choice of time horizon $u$, since $\left\{\pi_{i t}\right\}$ and $\left\{H_{t}\right\}$ are only defined for $t \in[0, u]$; but it is straightforward to see that (15) and (16) remain valid for all $t \in[0, \infty)$.

The arguments establishing the validity of (i), (ii), and (iii) above can be sketched briefly as follows. First we note that the relation between expectation under the $\mathbb{P}$-measure and expectation under the $\mathbb{Q}$-measure is given by

$$
\mathbb{E}^{\mathbb{Q}}[X]=\mathbb{E}^{\mathbb{P}}\left[\Lambda_{u}^{-1} X\right] .
$$

To see that $\left\{\xi_{t}\right\}_{0 \leq t<\infty}$ is a Brownian motion on $(\Omega, \mathcal{F}, \mathbb{Q})$ it suffices to show that

$$
\mathbb{E}^{\mathbb{Q}}\left[\mathrm{e}^{x \xi_{s}+y \xi_{t}}\right]=\mathrm{e}^{\frac{1}{2}\left(x^{2} s+y^{2} t+2 x y s\right)}
$$

for $s \leq t$ and for all $x, y$. In particular, if $\left\{\xi_{t}\right\}$ possesses this bi-characteristic function, then it follows at once that $\left\{\xi_{t}\right\}$ is $\mathbb{Q}$-Gaussian, and that $\operatorname{Cov}\left[\xi_{s}, \xi_{t}\right]=s$ for $s \leq t$. These properties, together with the fact that $\left\{\xi_{t}\right\}$ is continuous, are sufficient to characterise it as a Brownian motion under $\mathbb{Q}$. The verification of (18) follows by a calculation that makes use of (10), (12), and (17), the $\mathbb{P}$-independence of $H$ and $\left\{B_{t}\right\}$, and basic properties of $\left\{B_{t}\right\}$ under $\mathbb{P}$. The $\mathbb{Q}$-independence of $H$ and $\left\{\xi_{t}\right\}$ then follows by a similar calculation that establishes that

$$
\mathbb{E}^{\mathbb{Q}}\left[\mathrm{e}^{x \xi_{t}+y H}\right]=\mathrm{e}^{\frac{1}{2} x^{2} t} \sum_{i} \pi_{i} \mathrm{e}^{y E_{i}} .
$$

To show that the probability law of $H$ is the same under $\mathbb{P}$ and $\mathbb{Q}$ we make use of the $\mathbb{P}$-independence of $H$ and $\left\{B_{t}\right\}$ to observe that

$$
\begin{aligned}
\mathbb{Q}\left(H=E_{i}\right) & =\mathbb{E}^{\mathbb{P}}\left[\Lambda_{u}^{-1} \mathbf{1}_{\left\{H=E_{i}\right\}}\right] \\
& =\mathbb{E}^{\mathbb{P}}\left[\mathbf{1}_{\left\{H=E_{i}\right\}} \exp \left(-H \int_{0}^{t} \sigma_{s} \mathrm{~d} B_{s}-\frac{1}{2} H^{2} \int_{0}^{t} \sigma_{s}^{2} \mathrm{~d} s\right)\right] \\
& =\mathbb{E}^{\mathbb{P}}\left[\mathbf{1}_{\left\{H=E_{i}\right\}} \exp \left(-E_{i} \int_{0}^{t} \sigma_{s} \mathrm{~d} B_{s}-\frac{1}{2} E_{i}^{2} \int_{0}^{t} \sigma_{s}^{2} \mathrm{~d} s\right)\right] \\
& =\mathbb{E}^{\mathbb{P}}\left[\mathbf{1}_{\left\{H=E_{i}\right\}}\right] \mathbb{E}^{\mathbb{P}}\left[\exp \left(-E_{i} \int_{0}^{t} \sigma_{s} \mathrm{~d} B_{s}-\frac{1}{2} E_{i}^{2} \int_{0}^{t} \sigma_{s}^{2} \mathrm{~d} s\right)\right] \\
& =\mathbb{P}\left(H=E_{i}\right) .
\end{aligned}
$$

As for (iii), we remark first that relation (14) is a special case of a more general result referred to as the Kallianpur-Striebel formula [27]. A derivation of (14) can be sketched as follows. We reverse the construction above and start with a probability space $(\Omega, \mathcal{F}, \mathbb{Q})$ on which $\left\{\xi_{t}\right\}_{0 \leq t<\infty}$ is a standard Brownian motion, and $H$ an independent random variable taking the values $\left\{E_{i}\right\}_{i=1,2, \ldots, N}$ with the probabilities $\left\{\pi_{i}\right\}_{i=1,2, \ldots, N}$. We fix a time interval $[0, u]$ and let $\mathcal{G}_{u}$ denote the $\sigma$-subalgebra of events generated by $\left\{\xi_{t}\right\}_{0 \leq t \leq u}$ and $H$. Assuming 
that $\left\{\sigma_{t}\right\}$ satisfies (15), we define the process $\left\{\Lambda_{t}\right\}_{0 \leq t \leq u}$ by (11), as before, and we introduce the measure $\mathbb{P}$ by setting $\mathrm{d} \mathbb{P}=\Lambda_{u} \mathrm{~d} \mathbb{Q}$, or equivalently, $\mathbb{P}(A)=\mathbb{E}^{\mathbb{Q}}\left[\Lambda_{u} \mathbf{1}_{\{\omega \in A\}}\right]$. Then the process $\left\{B_{t}\right\}_{0 \leq t \leq u}$ defined by $B_{t}=\xi_{t}-H \int_{0}^{t} \sigma_{s} \mathrm{~d} s$ is a Brownian motion with respect to $\mathbb{P}$, and is $\mathbb{P}$-independent of $H$. It is worthwhile emphasising that $\mathcal{F}_{t}=\sigma\left(\left\{\xi_{s}\right\}_{0 \leq s \leq t}\right)$, whereas $\mathcal{G}_{t}=\sigma\left(H,\left\{\xi_{s}\right\}_{0 \leq s \leq t}\right)=\sigma\left(H,\left\{B_{s}\right\}_{0 \leq s \leq t}\right)$. Thus $\mathcal{F}_{t} \subset \mathcal{G}_{t}$. One can think of $\left\{\mathcal{F}_{t}\right\}$ as the filtration generated by the dynamics of the state vector process $\left\{\left|\psi_{t}\right\rangle\right\}_{0 \leq t<\infty}$; whereas in the larger "nonphysical" filtration $\left\{\mathcal{G}_{t}\right\}$ the value of $H$ is already "known" at time 0 . It follows by the conditional form of the change of measure relation that

$$
\mathbb{E}^{\mathbb{P}}\left[f(H) \mid\left\{\xi_{s}\right\}_{0 \leq s \leq t}\right]=\frac{\mathbb{E}^{\mathbb{Q}}\left[f(H) \Lambda_{u} \mid\left\{\xi_{s}\right\}_{0 \leq s \leq t}\right]}{\mathbb{E}^{\mathbb{Q}}\left[\Lambda_{u} \mid\left\{\xi_{s}\right\}_{0 \leq s \leq t}\right]}
$$

Finally, we use the fact that $H$ and $\left\{\xi_{s}\right\}_{0 \leq s \leq u}$ are $\mathbb{Q}$-independent to deduce that

$$
\mathbb{E}^{\mathbb{Q}}\left[f(H) \Lambda_{u} \mid\left\{\xi_{s}\right\}_{0 \leq s \leq t}\right]=\mathbb{E}^{\mathbb{Q}}\left[f(H) \Lambda_{t} \mid\left\{\xi_{s}\right\}_{0 \leq s \leq t}\right]
$$

for any choice of $f(H)$, and as a consequence we deduce (14).

\section{ON THE MARKOVIAN NATURE OF THE ENERGY PROCESS}

Before we verify that our expression (16) for the conditional expectation of $H$ agrees with the energy process (2), it will be useful to show that $\left\{H_{t}\right\}$, as defined by (16), has the Markov property. We note in particular that by virtue of (16) we have

$$
H_{t}=\frac{\sum_{i} \pi_{i} E_{i} \exp \left(E_{i} \eta_{t}-\frac{1}{2} E_{i}^{2} \int_{0}^{t} \sigma_{s}^{2} \mathrm{~d} s\right)}{\sum_{i} \pi_{i} \exp \left(E_{i} \eta_{t}-\frac{1}{2} E_{i}^{2} \int_{0}^{t} \sigma_{s}^{2} \mathrm{~d} s\right)}
$$

where the process $\left\{\eta_{t}\right\}_{0 \leq t<\infty}$ is defined by

$$
\eta_{t}=\int_{0}^{t} \sigma_{s} \mathrm{~d} \xi_{s}
$$

To show that $\left\{H_{t}\right\}$ is a Markov process it will suffice if we can show: (i) that $\left\{\eta_{t}\right\}$ is a Markov process; and (ii) that $\eta_{t}$ can be expressed as a function of $H_{t}$, i.e. that the relation between $\eta_{t}$ and $H_{t}$ is invertible. In particular, if the relation between $\eta_{t}$ and $H$ is invertible, then the filtration generated by $\left\{\eta_{t}\right\}$ is the same as the filtration generated by $\left\{H_{t}\right\}$.

To verify that $\left\{\eta_{t}\right\}$ is Markovian we must demonstrate that, for all $T \geq t$ and for all $x \in \mathbb{R}$, we have

$$
\mathbb{P}\left(\eta_{T} \leq x \mid\left\{\eta_{s}\right\}_{0 \leq s \leq t}\right)=\mathbb{P}\left(\eta_{T} \leq x \mid \eta_{t}\right) .
$$

Alternatively, it suffices to show that

$$
\mathbb{P}\left(\eta_{t} \leq x \mid \eta_{s}, \eta_{s_{1}}, \eta_{s_{2}}, \ldots, \eta_{s_{k}}\right)=\mathbb{P}\left(\eta_{t} \leq x \mid \eta_{s}\right)
$$

for any collection of times $t, s, s_{1}, s_{2}, \ldots, s_{k}$ such that $t \geq s \geq s_{1} \geq s_{2} \geq \cdots \geq s_{k}>0$. To check that these conditions are satisfied we proceed as follows. First we note that by virtue of (24) we have

$$
\eta_{t}=H \int_{0}^{t} \sigma_{s}^{2} \mathrm{~d} s+\int_{0}^{t} \sigma_{s} \mathrm{~d} B_{s}
$$


and therefore

$$
\frac{\eta_{u}}{\int_{0}^{u} \sigma_{s}^{2} \mathrm{~d} s}-\frac{\eta_{v}}{\int_{0}^{v} \sigma_{s}^{2} \mathrm{~d} s}=\frac{\int_{0}^{u} \sigma_{s} \mathrm{~d} B_{s}}{\int_{0}^{u} \sigma_{s}^{2} \mathrm{~d} s}-\frac{\int_{0}^{v} \sigma_{s} \mathrm{~d} B_{s}}{\int_{0}^{v} \sigma_{s}^{2} \mathrm{~d} s}
$$

for all $u \geq v>0$. We shall establish that the process $\left\{\varphi_{u}\right\}_{u>0}$ defined by

$$
\varphi_{u}=\frac{\int_{0}^{u} \sigma_{s} \mathrm{~d} B_{s}}{\int_{0}^{u} \sigma_{s}^{2} \mathrm{~d} s}
$$

appearing in (28) has independent increments. In particular, since $\left\{\varphi_{u}\right\}$ is a Gaussian process it suffices to show that $\varphi_{b}-\varphi_{a}$ and $\varphi_{d}-\varphi_{c}$ are independent for all $d \geq c \geq b \geq a>0$. To see this, we note that since $\varphi_{b}-\varphi_{a}$ and $\varphi_{d}-\varphi_{c}$ are Gaussian random variables, for their independence it is sufficient to verify that the covariance $\mathbb{E}\left[\left(\varphi_{b}-\varphi_{a}\right)\left(\varphi_{d}-\varphi_{c}\right)\right]$ vanishes. But this follows after a short calculation making use of the Wiener-Ito isometry

$$
\mathbb{E}\left[\left(\int_{0}^{u} \sigma_{s} \mathrm{~d} B_{s}\right)\left(\int_{0}^{v} \sigma_{s} \mathrm{~d} B_{s}\right)\right]=\int_{0}^{u \wedge v} \sigma_{s}^{2} \mathrm{~d} s,
$$

where $u \wedge v=\min (u, v)$. Similarly, one can verify that $\varphi_{c}$ is independent of $\varphi_{b}-\varphi_{a}$ for all $c \geq b \geq a>0$. This follows from the fact that the increment $\varphi_{\infty}-\varphi_{c}$ is independent of $\varphi_{b}-\varphi_{a}$. Next we observe that as a consequence of the definitions of $\left\{\eta_{t}\right\}$ and $\left\{\varphi_{t}\right\}$ we have

$$
\mathbb{P}\left(\eta_{t} \leq x \mid \eta_{s}, \eta_{s_{1}}, \eta_{s_{2}}, \ldots, \eta_{s_{k}}\right)=\mathbb{P}\left(\eta_{t} \leq x \mid \eta_{s}, \varphi_{s}-\varphi_{s_{1}}, \varphi_{s_{1}}-\varphi_{s_{2}}, \ldots, \varphi_{s_{k-1}}-\varphi_{s_{k}}\right) .
$$

However, since $\eta_{t}$ and $\eta_{s}$ are independent of $\varphi_{s}-\varphi_{s_{1}}, \varphi_{s_{1}}-\varphi_{s_{2}}, \ldots, \varphi_{s_{k-1}}-\varphi_{s_{k}}$, the desired result (26) follows.

To show that $H_{t}$ is invertible as a function of $\eta_{t}$ it will suffice to show that for each fixed $t$ the function

$$
H(\eta, t)=\frac{\sum_{i} \pi_{i} E_{i} \exp \left(E_{i} \eta-\frac{1}{2} E_{i}^{2} \int_{0}^{t} \sigma_{s}^{2} \mathrm{~d} s\right)}{\sum_{i} \pi_{i} \exp \left(E_{i} \eta-\frac{1}{2} E_{i}^{2} \int_{0}^{t} \sigma_{s}^{2} \mathrm{~d} s\right)}
$$

is monotonic in $\eta$. But this can be seen immediately, since

$$
\frac{\partial H(\eta, t)}{\partial \eta}=\frac{\sum_{i} \pi_{i}\left(E_{i}-H(\eta, t)\right)^{2} \exp \left(E_{i} \eta-\frac{1}{2} E_{i}^{2} \int_{0}^{t} \sigma_{s}^{2} \mathrm{~d} s\right)}{\sum_{i} \pi_{i} \exp \left(E_{i} \eta-\frac{1}{2} E_{i}^{2} \int_{0}^{t} \sigma_{s}^{2} \mathrm{~d} s\right)},
$$

which is positive for all values of $\eta$. As a consequence we see that the process $\left\{H_{t}\right\}$ as defined by (16) has the Markov property. In particular, we have the following equalities: $\mathbb{P}\left(H_{T} \leq x \mid\left\{H_{s}\right\}_{0 \leq s \leq t}\right)=\mathbb{P}\left(H_{T} \leq x \mid H_{t}\right)=\mathbb{P}\left(H_{T} \leq x \mid \eta_{t}\right)=\mathbb{P}\left(H_{T} \leq x \mid \mathcal{F}_{t}\right)$.

It is interesting to note that the conditional probability process $\left\{\pi_{i t}\right\}$ given by (15) can be expressed in the form

$$
\pi_{i t}=\frac{\pi_{i s} \exp \left(E_{i} \int_{s}^{t} \sigma_{u} \mathrm{~d} \xi_{u}-\frac{1}{2} E_{i}^{2} \int_{s}^{t} \sigma_{u}^{2} \mathrm{~d} u\right)}{\sum_{i} \pi_{i s} \exp \left(E_{i} \int_{s}^{t} \sigma_{u} \mathrm{~d} \xi_{u}-\frac{1}{2} E_{i}^{2} \int_{s}^{t} \sigma_{u}^{2} \mathrm{~d} u\right)} .
$$


The interpretation of this relation is that the energy-based reduction models exhibit a fundamental dynamic consistency property. In other words, if at some intermediate time $s$, where $0<s<t$, we take note of the a posteriori conditional probability $\pi_{i s}$, which is based on all information available up to time $s$, then we see that the resulting "new" model for the collapse of the wave function, given by (34), is of exactly the same form as the original model, with $\pi_{i s}$ playing the role of the new a priori probability. This means that the choice of the initial time $t=0$ has no preferential status in the theory. Indeed, the dynamical consistency of the energy-based reduction theory shows that the objections raised by Pearle 32] in this connection are groundless. It is perfectly consistent to regard the collapse process as having already started at some earlier time than "the present".

\section{INNOVATION PROCESS AND SOLUTION}

In Section III we calculated the expectation of the random variable $H$ conditional on the specification of $\left\{\xi_{s}\right\}_{0 \leq s \leq t}$, and we claimed that the result gives the energy expectation process (2). The aim of this section is to verify this claim, by showing how $\left\{\xi_{t}\right\}$ is related to the Brownian motion $\left\{W_{t}\right\}$ of equation (11). We begin by analysing the dynamics of the energy process (16). A direct application of Ito's rule shows that

$$
\mathrm{d} H_{t}=-\sigma_{t}^{2} H_{t} V_{t} \mathrm{~d} t+\sigma_{t} V_{t} \mathrm{~d} \xi_{t}
$$

where

$$
V_{t}=\mathbb{E}\left[\left(H-H_{t}\right)^{2} \mid\left\{\xi_{s}\right\}_{0 \leq s \leq t}\right]
$$

is the conditional variance of $H$. The next step is to define a random process $\left\{W_{t}\right\}$ by

$$
W_{t}=\xi_{t}-\int_{0}^{t} \sigma_{s} H_{s} \mathrm{~d} s
$$

It follows then from (35) that the dynamical equation for $\left\{H_{t}\right\}$ is given by:

$$
\mathrm{d} H_{t}=\sigma_{t} V_{t} \mathrm{~d} W_{t} .
$$

Equation (38) can be given a simple heuristic interpretation if we write it in the form

$$
H_{t+\mathrm{d} t}-\mathbb{E}\left[H_{t+\mathrm{d} t} \mid\left\{\xi_{s}\right\}_{0 \leq s \leq t}\right]=\sigma_{t} V_{t} \mathrm{~d} W_{t} .
$$

Since at $t$ the variance $V_{t}$ is known, as is also the conditional expectation $\mathbb{E}\left[H_{t+\mathrm{d} t} \mid\left\{\xi_{s}\right\}_{0 \leq s \leq t}\right]$, we see that $\mathrm{d} W_{t}$ embodies the "new information" entering the system between $t$ and $t+\mathrm{d} t$. It is for this reason that $\left\{W_{t}\right\}$ is called an innovation process.

We claim that $\left\{W_{t}\right\}$ is an $\left\{\mathcal{F}_{t}\right\}$-Brownian motion. Here we recall that $\left\{\mathcal{F}_{t}\right\}$ denotes the filtration generated by the process $\left\{\xi_{t}\right\}$. Thus, conditioning with respect to the $\sigma$-algebra $\mathcal{F}_{t}$ means conditioning with respect to the trajectory $\left\{\xi_{s}\right\}_{0 \leq s \leq t}$. To proceed we need now a more precise definition of Brownian motion. A process $\left\{W_{t}\right\}_{0 \leq t \leq \infty}$ on a probability space $(\Omega, \mathcal{F}, \mathbb{P})$ with filtration $\left\{\mathcal{F}_{t}\right\}_{0 \leq t \leq \infty}$ is said to be a standard Brownian motion if it satisfies the following properties: (i) $W_{0}=0$ almost surely; (ii) $\left\{W_{t}\right\}$ is $\left\{\mathcal{F}_{t}\right\}$-adapted; and (iii) for all $0 \leq s \leq t$ the increment $W_{t}-W_{s}$ is normally distributed with mean zero and variance $t-s$, and is independent of $\mathcal{F}_{s}$. In the present context we shall use the so-called Lévy's characterisation 
of Brownian motion, which states that if $\left\{W_{t}\right\}$ is a martingale, and if $\left(\mathrm{d} W_{t}\right)^{2}=\mathrm{d} t$, then $\left\{W_{t}\right\}$ is a Brownian motion.

Let us consider the martingale condition. Writing $\mathbb{E}_{t}[-]=\mathbb{E}\left[-\mid \mathcal{F}_{t}\right]$ for conditional expectation with respect to $\mathcal{F}_{t}$, we shall establish that $\mathbb{E}_{t}\left[W_{T}\right]=W_{t}$ for $t \leq T$. We find

$$
\begin{aligned}
\mathbb{E}_{t}\left[W_{T}\right] & =\mathbb{E}_{t}\left[\xi_{T}\right]-\mathbb{E}_{t}\left[\int_{0}^{T} \sigma_{s} H_{s} \mathrm{~d} s\right] \\
& =H_{t} \int_{0}^{T} \sigma_{s} \mathrm{~d} s+\mathbb{E}_{t}\left[B_{T}\right]-\int_{0}^{T} \sigma_{s} \mathbb{E}_{t}\left[H_{s}\right] \mathrm{d} s,
\end{aligned}
$$

where we have substituted (44) and we have interchanged the order of integration and expectation by use of the Fubini theorem. Next, we note that

$$
\begin{aligned}
\int_{0}^{T} \sigma_{s} \mathbb{E}_{t}\left[H_{s}\right] \mathrm{d} s & =\int_{0}^{t} \sigma_{s} \mathbb{E}_{t}\left[H_{s}\right] \mathrm{d} s+\int_{t}^{T} \sigma_{s} \mathbb{E}_{t}\left[H_{s}\right] \mathrm{d} s \\
& =\int_{0}^{t} \sigma_{s} H_{s} \mathrm{~d} s+H_{t} \int_{t}^{T} \sigma_{s} \mathrm{~d} s .
\end{aligned}
$$

Here we have used the fact that $H_{t}=\mathbb{E}_{t}[H]$ satisfies the martingale condition $\mathbb{E}_{t}\left[H_{s}\right]=H_{t}$ for $s \geq t$. Hence substituting (41) into (40) we obtain

$$
\mathbb{E}_{t}\left[W_{T}\right]=H_{t} \int_{0}^{t} \sigma_{s} \mathrm{~d} s+\mathbb{E}_{t}\left[B_{T}\right]-\int_{0}^{t} \sigma_{s} H_{s} \mathrm{~d} s .
$$

Finally, from the tower property of conditional expectation we have

$$
\mathbb{E}_{t}\left[B_{T}\right]=\mathbb{E}_{t}\left[\mathbb{E}\left[B_{T} \mid\left\{B_{s}\right\}_{0 \leq s \leq t}, H\right]\right]=\mathbb{E}_{t}\left[B_{t}\right]
$$

Inserting this relation into (42) we obtain

$$
\begin{aligned}
\mathbb{E}_{t}\left[W_{T}\right] & =\mathbb{E}_{t}\left[H \int_{0}^{t} \sigma_{s} \mathrm{~d} s+B_{t}\right]-\int_{0}^{t} \sigma_{s} H_{s} \mathrm{~d} s \\
& =\xi_{t}-\int_{0}^{t} \sigma_{s} H_{s} \mathrm{~d} s=W_{t}
\end{aligned}
$$

where we have used the relation $\mathbb{E}_{t}\left[\xi_{t}\right]=\xi_{t}$. This establishes that $\left\{W_{t}\right\}$ is an $\left\{\mathcal{F}_{t}\right\}$-martingale. On the other hand, because

$$
\mathrm{d} W_{t}=\left(H-H_{t}\right) \sigma_{t} \mathrm{~d} t+\mathrm{d} B_{t}
$$

it follows that $\left(\mathrm{d} W_{t}\right)^{2}=\mathrm{d} t$. Taking this together with the fact that $\left\{W_{t}\right\}$ is an $\left\{\mathcal{F}_{t}\right\}$ martingale, we conclude by Lévy's criterion that $\left\{W_{t}\right\}$ is an $\left\{\mathcal{F}_{t}\right\}$-Brownian motion.

We are now closer to establishing the relation between (2) and (16). To this end we consider the conditional probability (15) that $H$ takes the value $E_{i}$. Taking the stochastic differential of (15) and substituting (37) into the result, we find, after some rearrangement, that $\left\{\pi_{i t}\right\}$ satisfies

$$
\mathrm{d} \pi_{i t}=\sigma_{t}\left(E_{i}-H_{t}\right) \pi_{i t} \mathrm{~d} W_{t}
$$


With another application of the Ito formula, we thus deduce that

$$
\mathrm{d} \pi_{i t}^{1 / 2}=-\frac{1}{8} \sigma_{t}^{2}\left(E_{i}-H_{t}\right)^{2} \pi_{i t}^{1 / 2} \mathrm{~d} t+\frac{1}{2} \sigma_{t}\left(E_{i}-H_{t}\right) \pi_{i t}^{1 / 2} \mathrm{~d} W_{t} .
$$

Finally, if we let $\left|\phi_{i}\right\rangle$ denote the normalised Lüders state [4, 30] associated with the eigenvalue $E_{i}$, and define $\left\{\left|\psi_{t}\right\rangle\right\}$ according to

$$
\left|\psi_{t}\right\rangle=\sum_{i} \mathrm{e}^{-\mathrm{i} E_{i} t} \pi_{i t}^{1 / 2}\left|\phi_{i}\right\rangle
$$

then it follows at once from (47) that $\left\{\left|\psi_{t}\right\rangle\right\}$ satisfies the time-dependent energy-based stochastic Schrödinger equation (1).

In summary, if we define $\left\{\xi_{t}\right\}$ and $\left\{\eta_{t}\right\}$ in terms of the independent random data $H$ and $\left\{B_{t}\right\}$ according to (44) and (24), and if we define $\left\{H_{t}\right\}$ and $\left\{\left|\psi_{t}\right\rangle\right\}$ by (16) and (48), and $\left\{W_{t}\right\}$ by (37), then $\left\{\left|\psi_{t}\right\rangle\right\}$ solves (11) for the given Hamiltonian $\hat{H}$ and initial condition $\left|\psi_{0}\right\rangle$.

\section{DIRECT DERIVATION OF INDEPENDENT RANDOM DATA}

The way in which we have solved equation (1) is by introducing the concept of a quantum information process (44) specified in terms of a random variable $H$ and an independent Brownian motion $\left\{B_{t}\right\}$. It is possible, however, to deduce the existence of these random data directly from (11). In this section we shall illustrate this reverse construction.

We begin by remarking that the energy-based stochastic Schrödinger equation can be cast into integral form, incorporating the initial condition $\left|\psi_{0}\right\rangle$, as follows:

$$
\left|\psi_{t}\right\rangle=\exp \left(-\mathrm{i} \hat{H} t-\frac{1}{4} \int_{0}^{t} \sigma_{s}^{2}\left(\hat{H}-H_{s}\right)^{2} \mathrm{~d} s+\frac{1}{2} \int_{0}^{t} \sigma_{s}\left(\hat{H}-H_{s}\right) \mathrm{d} W_{s}\right)\left|\psi_{0}\right\rangle
$$

After some simple rearrangement we then deduce that

$$
\left|\psi_{t}\right\rangle=\frac{\exp \left(-\mathrm{i} \hat{H} t+\frac{1}{2} \hat{H} \int_{0}^{t} \sigma_{s}\left(\mathrm{~d} W_{s}+\sigma_{s} H_{s} \mathrm{~d} s\right)-\frac{1}{4} \hat{H}^{2} \int_{0}^{t} \sigma_{s}^{2} \mathrm{~d} s\right)\left|\psi_{0}\right\rangle}{\exp \left(\frac{1}{2} \int_{0}^{t} \sigma_{s} H_{s}\left(\mathrm{~d} W_{s}+\sigma_{s} H_{s} \mathrm{~d} s\right)-\frac{1}{4} \int_{0}^{t} \sigma_{s}^{2} H_{s}^{2} \mathrm{~d} s\right)} .
$$

Given the $\left\{\mathcal{F}_{t}\right\}$-adapted Brownian motion $\left\{W_{t}\right\}$ and the energy expectation process $\left\{H_{t}\right\}=$ $\left\langle\psi_{t}|\hat{H}| \psi_{t}\right\rangle$ we now define a process $\left\{\xi_{t}\right\}$ by writing

$$
\xi_{t}=W_{t}+\int_{0}^{t} \sigma_{s} H_{s} \mathrm{~d} s .
$$

It follows then that $\left|\psi_{t}\right\rangle$ can be written in the form

$$
\left|\psi_{t}\right\rangle=\frac{\exp \left(-\mathrm{i} \hat{H} t+\frac{1}{2} \hat{H} \int_{0}^{t} \sigma_{s} \mathrm{~d} \xi_{t}-\frac{1}{4} \hat{H}^{2} \int_{0}^{t} \sigma_{s}^{2} \mathrm{~d} s\right)\left|\psi_{0}\right\rangle}{\exp \left(\frac{1}{2} \int_{0}^{t} \sigma_{s} H_{s} \mathrm{~d} \xi_{t}-\frac{1}{4} \int_{0}^{t} \sigma_{s}^{2} H_{s}^{2} \mathrm{~d} s\right)} .
$$

With these ingredients at hand we now claim the following: The random variables $H=$ $\lim _{t \rightarrow \infty} H_{t}$ and $B_{t}=\xi_{t}-H \int_{0}^{t} \sigma_{s} \mathrm{~d} s$ are independent. Furthermore, the process $\left\{B_{t}\right\}$ thus defined is a standard $\mathbb{P}$-Brownian motion. 
The existence of the random variable $H$ is ensured by the martingale convergence theorem. The fact that $H$ has the distribution $\mathbb{P}\left(H=E_{i}\right)=\pi_{i}$ then follows as a consequence of known properties of the stochastic equation (II). To show that $H$ and $B_{t}$ are independent (for any value of $t$ ) it suffices to show that

$$
\mathbb{E}^{\mathbb{P}}\left[\mathrm{e}^{x B_{t}+y H}\right]=\mathbb{E}^{\mathbb{P}}\left[\mathrm{e}^{x B_{t}}\right] \mathbb{E}^{\mathbb{P}}\left[\mathrm{e}^{y H}\right]
$$

for any $x, y$. The proof proceeds as follows. First, by use of the tower property of conditional expectation we have

$$
\begin{aligned}
\mathbb{E}^{\mathbb{P}}\left[\mathrm{e}^{x B_{t}+y H}\right] & =\mathbb{E}^{\mathbb{P}}\left[\mathrm{e}^{x\left(\xi_{t}-H \int_{0}^{t} \sigma_{s} \mathrm{~d} s\right)+y H}\right] \\
& =\mathbb{E}^{\mathbb{P}}\left[\mathrm{e}^{x \xi_{t}} \mathbb{E}_{t}^{\mathbb{P}}\left[\mathrm{e}^{\left(y-x \int_{0}^{t} \sigma_{s} \mathrm{~d} s\right) H}\right]\right],
\end{aligned}
$$

where $\mathbb{E}_{t}$ denotes conditional expectation with respect to $\mathcal{F}_{t}$. Here we have used the fact that $\xi_{t}$ is $\mathcal{F}_{t}$-measurable. For the inner expectation we can write

$$
\mathbb{E}_{t}^{\mathbb{P}}\left[\mathrm{e}^{\left(y-x \int_{0}^{t} \sigma_{s} \mathrm{~d} s\right) H}\right]=\sum_{i} \pi_{i t} \mathrm{e}^{\left(y-x \int_{0}^{t} \sigma_{s} \mathrm{~d} s\right) E_{i}},
$$

where $\pi_{i t}$ is defined as the conditional probability

$$
\pi_{i t}=\mathbb{P}\left(H=E_{i} \mid \mathcal{F}_{t}\right) .
$$

To obtain an expression for $\left\{\pi_{i t}\right\}$ we recall [4, 6] that since the projection operator $\hat{\Pi}_{i}$ commutes with the Hamiltonian, the bounded process $\left\{\left\langle\psi_{t}\left|\hat{\Pi}_{i}\right| \psi_{t}\right\rangle\right\}$ is a martingale. It follows that

$$
\left\langle\psi_{t}\left|\hat{\Pi}_{i}\right| \psi_{t}\right\rangle=\mathbb{E}_{t}^{\mathbb{P}}\left[\left\langle\psi_{\infty}\left|\hat{\Pi}_{i}\right| \psi_{\infty}\right\rangle\right] .
$$

On the other hand, by known properties of the reduction process (II) we have $\left\langle\psi_{\infty}\left|\hat{\Pi}_{i}\right| \psi_{\infty}\right\rangle=$ $\mathbf{1}_{\left\{H=E_{i}\right\}}$, and hence

$$
\left\langle\psi_{t}\left|\hat{\Pi}_{i}\right| \psi_{t}\right\rangle=\mathbb{E}_{t}^{\mathbb{P}}\left[\mathbf{1}_{\left\{H=E_{i}\right\}}\right] .
$$

We are therefore able to deduce that $\mathbb{P}\left(H=E_{i} \mid \mathcal{F}_{t}\right)=\pi_{i t}=\left\langle\psi_{t}\left|\hat{\Pi}_{i}\right| \psi_{t}\right\rangle$. A short calculation making use of (52) and properties of the projection operator then shows that

$$
\pi_{i t}=\frac{\pi_{i} \exp \left(E_{i} \int_{0}^{t} \sigma_{s} \mathrm{~d} \xi_{t}-\frac{1}{2} E_{i}^{2} \int_{0}^{t} \sigma_{s}^{2} \mathrm{~d} s\right)}{\exp \left(\int_{0}^{t} \sigma_{s} H_{s} \mathrm{~d} \xi_{t}-\frac{1}{2} \int_{0}^{t} \sigma_{s}^{2} H_{s}^{2} \mathrm{~d} s\right)} .
$$

In fact, with a little work one can also show that $\pi_{i t}=\left|\left\langle\phi_{i} \mid \psi_{t}\right\rangle\right|^{2}$. In other words, $\pi_{i t}$ is given by the usual formula for the quantum-mechanical transition probability from the state $\left|\psi_{t}\right\rangle$ to the Lüders state $\left|\phi_{i}\right\rangle$. Of course, in standard quantum mechanics it is an assumption that $\pi_{i t}$, when defined in this way, has the interpretation of a transition probability. But in the stochastic theory, we deduce this property. 
Returning to our calculation of the inner conditional expectation in formula (54) we see as a consequence of (59) that

$$
\mathbb{E}_{t}^{\mathbb{P}}\left[\mathrm{e}^{\left(y-x \int_{0}^{t} \sigma_{s} \mathrm{~d} s\right) H}\right]=\frac{\sum_{i} \pi_{i} \mathrm{e}^{\left(y-x \int_{0}^{t} \sigma_{s} \mathrm{~d} s\right) E_{i}} \mathrm{e}^{E_{i} \int_{0}^{t} \sigma_{s} \mathrm{~d} \xi_{t}-\frac{1}{2} E_{i}^{2} \int_{0}^{t} \sigma_{s}^{2} \mathrm{~d} s}}{\mathrm{e}^{\int_{0}^{t} \sigma_{s} H_{s} \mathrm{~d} \xi_{t}-\frac{1}{2} \int_{0}^{t} \sigma_{s}^{2} H_{s}^{2} \mathrm{~d} s}} .
$$

It follows that

$$
\begin{aligned}
\mathbb{E}^{\mathbb{P}}\left[\mathrm{e}^{x B_{t}+y H}\right] & =\mathbb{E}^{\mathbb{P}}\left[\frac{\mathrm{e}^{x \xi_{t}} \sum_{i} \pi_{i} \mathrm{e}^{\left(y-x \int_{0}^{t} \sigma_{s} \mathrm{~d} s\right) E_{i}} \mathrm{e}^{E_{i} \int_{0}^{t} \sigma_{s} \mathrm{~d} \xi_{t}-\frac{1}{2} E_{i}^{2} \int_{0}^{t} \sigma_{s}^{2} \mathrm{~d} s}}{\mathrm{e}^{\int_{0}^{t} \sigma_{s} H_{s} \mathrm{~d} \xi_{t}-\frac{1}{2} \int_{0}^{t} \sigma_{s}^{2} H_{s}^{2} \mathrm{~d} s}}\right] \\
& =\sum_{i} \pi_{i} \mathrm{e}^{\left(y-x \int_{0}^{t} \sigma_{s} \mathrm{~d} s\right) E_{i}} \mathbb{E}^{\mathbb{P}}\left[\frac{\mathrm{e}^{x \xi_{t}} \mathrm{e}^{E_{i} \int_{0}^{t} \sigma_{s} \mathrm{~d} \xi_{t}-\frac{1}{2} E_{i}^{2} \int_{0}^{t} \sigma_{s}^{2} \mathrm{~d} s}}{\mathrm{e}^{\int_{0}^{t} \sigma_{s} H_{s} \mathrm{~d} \xi_{t}-\frac{1}{2} \int_{0}^{t} \sigma_{s}^{2} H_{s}^{2} \mathrm{~d} s}}\right] .
\end{aligned}
$$

Next we observe that since $\mathrm{d} \xi_{t}=\mathrm{d} W_{t}+\sigma_{t} H_{t} \mathrm{~d} t$, the expectation appearing above can be written in the form

$$
\mathbb{E}^{\mathbb{P}}\left[\frac{\mathrm{e}^{x \xi_{t}} \mathrm{e}^{E_{i} \int_{0}^{t} \sigma_{s} \mathrm{~d} \xi_{t}-\frac{1}{2} E_{i}^{2} \int_{0}^{t} \sigma_{s}^{2} \mathrm{~d} s}}{\mathrm{e}^{\int_{0}^{t} \sigma_{s} H_{s} \mathrm{~d} \xi_{t}-\frac{1}{2} \int_{0}^{t} \sigma_{s}^{2} H_{s}^{2} \mathrm{~d} s}}\right]=\mathbb{E}^{\mathbb{P}}\left[\mathrm{e}^{-\int_{0}^{t} \sigma_{s} H_{s} \mathrm{~d} W_{s}-\frac{1}{2} \int_{0}^{t} \sigma_{s}^{2} H_{s}^{2} \mathrm{~d} s} \mathrm{e}^{x \xi_{t}} \mathrm{e}^{E_{i} \int_{0}^{t} \sigma_{s} \mathrm{~d} \xi_{t}-\frac{1}{2} E_{i}^{2} \int_{0}^{t} \sigma_{s}^{2} \mathrm{~d} s}\right] .
$$

However, the expression

$$
\Phi_{t}=\exp \left(-\int_{0}^{t} \sigma_{s} H_{s} \mathrm{~d} W_{t}-\frac{1}{2} \int_{0}^{t} \sigma_{s}^{2} H_{s}^{2} \mathrm{~d} s\right)
$$

is the change-of-measure density over the interval $[0, t]$ needed to make $\left\{\xi_{s}\right\}_{0 \leq s \leq t}$ a standard Brownian motion. Writing $\mathbb{Q}$ for the resulting new measure, by use of the Girsanov theorem we obtain

$$
\mathbb{E}^{\mathbb{P}}\left[\mathrm{e}^{x B_{t}+y H}\right]=\sum_{i} \pi_{i} \mathrm{e}^{\left(y-x \int_{0}^{t} \sigma_{s} \mathrm{~d} s\right) E_{i}} \mathbb{E}^{\mathbb{Q}}\left[\mathrm{e}^{x \xi_{t}} \mathrm{e}^{E_{i} \int_{0}^{t} \sigma_{s} \mathrm{~d} \xi_{t}-\frac{1}{2} E_{i}^{2} \int_{0}^{t} \sigma_{s}^{2} \mathrm{~d} s}\right] .
$$

By rearranging terms, the expectation on the right above can be rewritten in the form

$$
\mathbb{E}^{\mathbb{Q}}\left[\mathrm{e}^{x \xi_{t}} \mathrm{e}^{E_{i} \int_{0}^{t} \sigma_{s} \mathrm{~d} \xi_{t}-\frac{1}{2} E_{i}^{2} \int_{0}^{t} \sigma_{s}^{2} \mathrm{~d} s}\right]=\mathrm{e}^{\frac{1}{2} x^{2} t+x E_{i} \int_{0}^{t} \sigma_{s} \mathrm{~d} s} \mathbb{E}^{\mathbb{Q}}\left[\mathrm{e}^{\int_{0}^{t}\left(E_{i} \sigma_{s}+x\right) \mathrm{d} \xi_{t}-\frac{1}{2} \int_{0}^{t}\left(E_{i} \sigma_{s}+x\right)^{2} \mathrm{~d} s}\right] .
$$

Since $\left\{\xi_{t}\right\}$ is a $\mathbb{Q}$-Brownian motion, it follows that the expectation appearing on the right side of (65) has the value unity. As a consequence, we deduce that

$$
\mathbb{E}^{\mathbb{P}}\left[\mathrm{e}^{x B_{t}+y H}\right]=\mathrm{e}^{\frac{1}{2} x^{2} t} \sum_{i} \pi_{i} \mathrm{e}^{y E_{i}}
$$

We see therefore that $B_{t}$ and $H$ are independent, as claimed. We also see, by the form of its characteristic function, that $B_{t}$ is Gaussian, with mean 0 and variance $t$. An argument similar to that presented above shows that if $s \leq t$ then

$$
\mathbb{E}^{\mathbb{P}}\left[\mathrm{e}^{x B_{s}+y\left(B_{t}-B_{s}\right)}\right]=\mathrm{e}^{\frac{1}{2} x^{2} s} \mathrm{e}^{y^{2}(t-s)}
$$

for all $x, y$, and hence $\operatorname{Cov}\left(B_{s}, B_{t}\right)=s$ for $s \leq t$. Since $\left\{B_{t}\right\}$ is a continuous Gaussian process and has the correct mean and autocovariance properties, we deduce that $\left\{B_{t}\right\}$ is a standard $\mathbb{P}$-Brownian motion. 
We have therefore shown that the solution to (11) can be put into the form (52), with $\xi_{t}=H \int_{0}^{t} \sigma_{s} \mathrm{~d} s+B_{t}$, where $H=\lim _{t \rightarrow \infty}\left\langle\psi_{t}|\hat{H}| \psi_{t}\right\rangle$, and where $\left\{B_{t}\right\}$ is an independent standard Brownian motion.

Conversely, as we have shown earlier in the paper, if on a fixed probability space $(\Omega, \mathcal{F}, \mathbb{P})$ we are given a random variable $H$ with the distribution $\mathbb{P}\left(H=E_{i}\right)=\pi_{i}$ together with an independent standard Brownian motion $\left\{B_{t}\right\}$, then we can proceed as follows: we construct the process $\left\{\xi_{t}\right\}$ by setting $\xi_{t}=H \int_{0}^{t} \sigma_{s} \mathrm{~d} s+B_{t}$; we define the process $\left\{H_{t}\right\}$ by $H_{t}=$ $\mathbb{E}\left[H \mid \mathcal{F}_{t}\right]$ where $\left\{\mathcal{F}_{t}\right\}$ is the filtration generated by $\left\{\xi_{t}\right\}$; we define the process $\left\{\pi_{i t}\right\}$ by setting $\pi_{i t}=\mathbb{E}\left[\mathbf{1}_{\left\{H=E_{i}\right\}} \mid \mathcal{F}_{t}\right]$; and we define the $\left\{\mathcal{F}_{t}\right\}$-adapted Brownian motion $\left\{W_{t}\right\}$ by setting $W_{t}=\xi_{t}-\int_{0}^{t} \sigma_{s} H_{s} \mathrm{~d} s$. Then $\left\{\left|\psi_{t}\right\rangle\right\}$, defined by $\left|\psi_{t}\right\rangle=\sum_{i} \mathrm{e}^{-\mathrm{i} E_{i} t} \pi_{i t}^{1 / 2}\left|\phi_{i}\right\rangle$, satisfies (II).

Let us now consider briefly the "linearisation" technique (see, e.g., 8] and references cited therein) often used for studying the dynamics of (11). Starting with (11), we proceed as above to deduce (52), defining the process $\left\{\xi_{t}\right\}$ as in (51). Next we observe that if we introduce a process $\left\{\left|\Psi_{t}\right\rangle\right\}$ by defining

$$
\left|\Psi_{t}\right\rangle=\exp \left(-\mathrm{i} \hat{H} t+\frac{1}{2} \hat{H} \int_{0}^{t} \sigma_{s} \mathrm{~d} \xi_{t}-\frac{1}{4} \hat{H}^{2} \int_{0}^{t} \sigma_{s}^{2} \mathrm{~d} s\right)\left|\psi_{0}\right\rangle,
$$

then $\left|\Psi_{t}\right\rangle$ satisfies

$$
\mathrm{d}\left|\Psi_{t}\right\rangle=-\mathrm{i} \hat{H}\left|\Psi_{t}\right\rangle \mathrm{d} t-\frac{1}{8} \sigma_{t}^{2} \hat{H}^{2}\left|\Psi_{t}\right\rangle \mathrm{d} t+\frac{1}{2} \sigma_{t} \hat{H}\left|\Psi_{t}\right\rangle \mathrm{d} \xi_{t} .
$$

We note that $\left|\Psi_{t}\right\rangle$ appears in the numerator of (52). Thus the relation between $\left|\Psi_{t}\right\rangle$ and $\left|\psi_{t}\right\rangle$ is given by

$$
\left|\psi_{t}\right\rangle=\frac{\left|\Psi_{t}\right\rangle}{\sqrt{\left\langle\Psi_{t} \mid \Psi_{t}\right\rangle}}
$$

where

$$
\left\langle\Psi_{t} \mid \Psi_{t}\right\rangle=\exp \left(\int_{0}^{t} \sigma_{s} H_{s} \mathrm{~d} \xi_{t}-\frac{1}{2} \int_{0}^{t} \sigma_{s}^{2} H_{s}^{2} \mathrm{~d} s\right) .
$$

Now suppose we fix a finite time interval $[0, T]$, and change to a new measure $\mathbb{Q}$ by use of the density $\Phi_{T}=\left\langle\Psi_{T} \mid \Psi_{T}\right\rangle^{-1}$. Then over the interval $[0, T]$ the process $\left\{\xi_{t}\right\}$ is a $\mathbb{Q}$-Brownian motion. Thus in the measure $\mathbb{Q}$ we can "solve" (1D) by expressing $\left|\psi_{t}\right\rangle$ in terms of $\left|\Psi_{t}\right\rangle$, making use of (68) and (70). Equation (69) holds under both $\mathbb{P}$ and $\mathbb{Q}$, but under $\mathbb{Q}$ it is a linear equation, and hence under $\mathbb{Q}$ we can regard (68) as the solution of (69).

The existence of this underlying "linearisation" of (11), together with the fact that there is only a single Hermitian operator appearing in the dynamics, may help to explain why the problem is exactly solvable. Of course, the "physics" is in the measure $\mathbb{P}$, so any application of the linearisation technique to solve a physical model typically involves using the density $\Phi_{T}^{-1}$ to change from $\mathbb{Q}$ back to $\mathbb{P}$. In practical terms this means that realistic simulations of the trajectories of $\left|\psi_{t}\right\rangle$ cannot be efficiently achieved by use of the linearisation technique.

On the other hand, our "random data" method involves a construction that is carried out entirely in the physical measure. The auxiliary measures are introduced simply for the purpose of verifying the results, not for the actual specification of the solution. In that sense, the random data method can be regarded as a major improvement over the linearisation method. In particular, to simulate a set of trajectories for $\left\{\left|\psi_{t}\right\rangle\right\}$ we only need to simulate outcomes for $H$ and $\left\{B_{t}\right\}$ in the measure $\mathbb{P}$. 


\section{VERIFICATION OF COLLAPSE PROPERTY}

In this section we shall verify directly that the solution (48) of the stochastic Schrödinger equation (11) gives rise to the collapse of the wave function. By substituting (41) into (15), setting $H=E_{k}$, and inserting the resulting expression into (48), we can express the solution of (1), conditional on $H=E_{k}$ for some fixed value of $k$, in the form

$$
\left|\psi_{t}\right\rangle=\frac{\sum_{i} \sqrt{\pi_{i}} \exp \left(-\mathrm{i} E_{i} t+\frac{1}{2} E_{i} E_{k} \int_{0}^{t} \sigma_{s}^{2} \mathrm{~d} s+\frac{1}{2} E_{i} \int_{0}^{t} \sigma_{s} \mathrm{~d} B_{s}-\frac{1}{4} E_{i}^{2} \int_{0}^{t} \sigma_{s}^{2} \mathrm{~d} s\right)\left|\phi_{i}\right\rangle}{\left[\sum_{i} \pi_{i} \exp \left(E_{i} E_{k} \int_{0}^{t} \sigma_{s}^{2} \mathrm{~d} s+E_{i} \int_{0}^{t} \sigma_{s} \mathrm{~d} B_{s}-\frac{1}{2} E_{i}^{2} \int_{0}^{t} \sigma_{s}^{2} \mathrm{~d} s\right)\right]^{1 / 2}} .
$$

In this situation we imagine that nature has "secretly" chosen the outcome $H=E_{k}$ (i.e. $H$ takes this value for the given $\omega \in \Omega$ ), and we want to show that the wave function evolves to the appropriate eigenstate. If we multiply the numerator and denominator of (172) by $\exp \left(-\frac{1}{4} E_{k}^{2} \int_{0}^{t} \sigma_{s}^{2} \mathrm{~d} s-\frac{1}{2} E_{k} \int_{0}^{t} \sigma_{s} \mathrm{~d} B_{s}\right)$ and write $\omega_{i k}=E_{i}-E_{k}$, then (72) becomes

$$
\begin{aligned}
\left|\psi_{t}\right\rangle & =\frac{\sum_{i} \sqrt{\pi_{i}} \exp \left(-\mathrm{i} E_{i} t-\frac{1}{4} \omega_{i k}^{2} \int_{0}^{t} \sigma_{s}^{2} \mathrm{~d} s+\frac{1}{2} \omega_{i k} \int_{0}^{t} \sigma_{s} \mathrm{~d} B_{s}\right)\left|\phi_{i}\right\rangle}{\left[\sum_{i} \pi_{i} \exp \left(-\frac{1}{2} \omega_{i k}^{2} \int_{0}^{t} \sigma_{s}^{2} \mathrm{~d} s+\omega_{i k} \int_{0}^{t} \sigma_{s} \mathrm{~d} B_{s}\right)\right]^{1 / 2}} \\
& =\frac{\sqrt{\pi_{k}} \mathrm{e}^{-\mathrm{i} E_{k} t}\left|\phi_{k}\right\rangle+\sum_{i \neq k} \sqrt{\pi_{i}} \exp \left(-\mathrm{i} E_{i} t-\frac{1}{4} \omega_{i k}^{2} \int_{0}^{t} \sigma_{s}^{2} \mathrm{~d} s+\frac{1}{2} \omega_{i k} \int_{0}^{t} \sigma_{s} \mathrm{~d} B_{s}\right)\left|\phi_{i}\right\rangle}{\left[\pi_{k}+\sum_{i \neq k} \pi_{i} \exp \left(-\frac{1}{2} \omega_{i k}^{2} \int_{0}^{t} \sigma_{s}^{2} \mathrm{~d} s+\omega_{i k} \int_{0}^{t} \sigma_{s} \mathrm{~d} B_{s}\right)\right]^{1 / 2}}
\end{aligned}
$$

It should be evident then, on account of condition (6), that $\left|\psi_{t}\right\rangle \rightarrow \mathrm{e}^{-\mathrm{i} E_{k} t}\left|\phi_{k}\right\rangle$ as $t \rightarrow \infty$. More precisely, defining

$$
M_{t}=\exp \left(\frac{1}{2} \omega \int_{0}^{t} \sigma_{s} \mathrm{~d} B_{s}-\frac{1}{4} \omega^{2} \int_{0}^{t} \sigma_{s}^{2} \mathrm{~d} s\right),
$$

we have, for any $\epsilon>0$,

$$
\begin{aligned}
\mathbb{P}\left(M_{t}>\epsilon\right) & =\mathbb{P}\left(\frac{1}{2} \omega \int_{0}^{t} \sigma_{s} \mathrm{~d} B_{s}-\frac{1}{4} \omega^{2} \int_{0}^{t} \sigma_{s}^{2} \mathrm{~d} s>\ln \epsilon\right) \\
& =\mathbb{P}\left(\frac{\int_{0}^{t} \sigma_{s} \mathrm{~d} B_{s}}{\sqrt{\int_{0}^{t} \sigma_{s}^{2} \mathrm{~d} s}}>\frac{1}{2} \omega \sqrt{\left.\int_{0}^{t} \sigma_{s}^{2} \mathrm{~d} s+\frac{2 \ln \epsilon}{\omega \sqrt{\int_{0}^{t} \sigma_{s}^{2} \mathrm{~d} s}}\right)}\right. \\
& =\left\{\begin{array}{l}
1-N\left(\frac{1}{2} \omega \sqrt{\int_{0}^{t} \sigma_{s}^{2} \mathrm{~d} s}+\frac{2 \ln \epsilon}{\omega \sqrt{\int_{0}^{t} \sigma_{s}^{2} \mathrm{~d} s}}\right)(\omega>0) \\
N\left(-\frac{1}{2} \omega \sqrt{\int_{0}^{t} \sigma_{s}^{2} \mathrm{~d} s}-\frac{2 \ln \epsilon}{\omega \sqrt{\int_{0}^{t} \sigma_{s}^{2} \mathrm{~d} s}}\right)
\end{array}\right.
\end{aligned}
$$

where $N(x)$ is the standard normal distribution function

$$
N(x)=\frac{1}{\sqrt{2 \pi}} \int_{-\infty}^{x} \mathrm{e}^{-\frac{1}{2} y^{2}} \mathrm{~d} y
$$


Here we have used the fact that $\int_{0}^{t} \sigma_{s} \mathrm{~d} B_{s} /\left(\int_{0}^{t} \sigma_{s}^{2} \mathrm{~d} s\right)^{1 / 2}$ is normally distributed with mean zero and variance unity. We thus see that (6) is satisfied if and only if $\mathbb{P}\left(M_{t}>\epsilon\right) \rightarrow 0$ as $t \rightarrow \infty$; and hence it follows that, given condition (6), the state vector collapses to the designated eigenstate. The intuition behind this result is that the "signal" component of $\left\{\eta_{t}\right\}$ eventually dominates over the "noise" component if (6) is satisfied. This is because the magnitude of $\int_{0}^{t} \sigma_{s} \mathrm{~d} B_{s}$ is on average about $\left(\int_{0}^{t} \sigma_{s}^{2} \mathrm{~d} s\right)^{1 / 2}$.

We note, incidentally, that if the leading order behaviour of the integral of $\left\{\sigma_{t}^{2}\right\}$ is such that $\int_{0}^{t} \sigma_{s}^{2} \mathrm{~d} s \sim t^{\alpha}$, with $\alpha>0$, then to leading order we have $\sigma_{t} \sim t^{\frac{1}{2}(\alpha-1)}$, and hence $\int_{0}^{t} \sigma_{s} \mathrm{~d} s \sim t^{\frac{1}{2}(\alpha+1)}$. Since the magnitude of $B_{t}$ is on average of the order $t^{1 / 2}$, we see that in this situation the signal component in (4) also dominates over the noise component.

\section{REDUCTION WITHOUT COMPLETE COLLAPSE}

In this section we show that when condition (6) is not satisfied, state reduction nevertheless takes place, in the sense that the energy variance decreases on average. However, unlike the models for which (6) is satisfied, in this case the terminal energy variance in general does not vanish. In other words, the state approaches an energy eigenstate, but does not get there. Physically, this situation corresponds to an approximate measurement of energy, in which some information concerning the energy of the system is revealed, but no definite outcome is obtained.

To analyse this situation we consider the energy variance process (36), which is given, equivalently, by

$$
V_{t}=\left\langle\psi_{t}\left|\hat{H}^{2}\right| \psi_{t}\right\rangle-\left\langle\psi_{t}|\hat{H}| \psi_{t}\right\rangle^{2}
$$

Taking the stochastic differential of (177) and using the dynamical equation (11) we find that

$$
\mathrm{d} V_{t}=-\sigma_{t}^{2} V_{t}^{2} \mathrm{~d} t+\sigma_{t} \kappa_{t} \mathrm{~d} W_{t},
$$

where $\kappa_{t}$ is the third central moment of the energy:

$$
\kappa_{t}=\left\langle\psi_{t}\left|\left(\hat{H}-H_{t}\right)^{3}\right| \psi_{t}\right\rangle
$$

We observe that the drift of $\left\{V_{t}\right\}$ is strictly negative. Therefore, the energy variance is on average decreasing. However, if $\left\{\sigma_{t}\right\}$ is a square-integrable function, then $\left\{V_{t}\right\}$ may converge to some finite nonzero value smaller than the initial value $V_{0}$. To investigate this scenario we write (78) in integral form:

$$
V_{t}=V_{0}-\int_{0}^{t} \sigma_{s}^{2} V_{s}^{2} \mathrm{~d} s+\int_{0}^{t} \sigma_{s} \kappa_{s} \mathrm{~d} W_{s} .
$$

Taking the expectation of each side, we obtain

$$
\mathbb{E}\left[V_{t}\right]=V_{0}-\int_{0}^{t} \sigma_{s}^{2} \mathbb{E}\left[V_{s}^{2}\right] \mathrm{d} s .
$$

On account of Jensen's inequality we have $\mathbb{E}\left[V_{t}^{2}\right] \geq\left(\mathbb{E}\left[V_{t}\right]\right)^{2}$, and hence (81) implies that

$$
\mathbb{E}\left[V_{t}\right] \leq V_{0}-\int_{0}^{t} \sigma_{s}^{2}\left(\mathbb{E}\left[V_{s}\right]\right)^{2} \mathrm{~d} s .
$$


Bearing in mind the fact that $\mathbb{E}\left[V_{t}\right] \leq \mathbb{E}\left[V_{s}\right]$ for $t \geq s$, the inequality (82) implies

$$
\mathbb{E}\left[V_{t}\right] \leq V_{0}-\left(\mathbb{E}\left[V_{t}\right]\right)^{2} \int_{0}^{t} \sigma_{s}^{2} \mathrm{~d} s .
$$

As a consequence, we obtain an upper bound on the expected value of the energy variance:

$$
\mathbb{E}\left[V_{t}\right] \leq \frac{1}{2 \int_{0}^{t} \sigma_{s}^{2} \mathrm{~d} s}\left(-1+\sqrt{1+4 V_{0} \int_{0}^{t} \sigma_{s}^{2} \mathrm{~d} s}\right) .
$$

In the limit $t \rightarrow \infty$ the inequality (84) determines an upper bound for the asymptotic value of the expected energy variance. That is, on average the energy variance will be reduced to a value no greater than the asymptotic value of the right side of (84). In particular, if (6) is satisfied, then $\mathbb{E}\left[V_{t}\right] \rightarrow 0$ as $t \rightarrow \infty$. On the other hand, if (6) is not satisfied, then we can obtain a lower bound for $\mathbb{E}\left[V_{\infty}\right]$. Let $V_{\max }$ denote the maximum possible variance that the energy can have, over all states. Then from (81) we get

$$
\mathbb{E}\left[V_{\infty}\right] \geq V_{0}-V_{\max }^{2} \int_{0}^{\infty} \sigma_{s}^{2} \mathrm{~d} s .
$$

Hence providing $\int_{0}^{\infty} \sigma_{s}^{2} \mathrm{~d} s<V_{0} / V_{\max }^{2}$ we are ensured that state reduction will be incomplete.

\section{FINITE-TIME COLLAPSE}

Having investigated the case in which the coupling $\left\{\sigma_{t}\right\}$ decays too rapidly to lead to a complete collapse of the wave function, we turn to the situation where the integral of the coupling $\left\{\sigma_{t}\right\}$ diverges over a finite time horizon. In particular, we consider the example

$$
\sigma_{t}=\frac{\sigma T}{T-t}
$$

where $\sigma>0$ and $T>0$ are fixed constants. For the resulting state vector dynamics we have the following stochastic differential equation:

$$
\mathrm{d}\left|\psi_{t}\right\rangle=-\mathrm{i} \hat{H}\left|\psi_{t}\right\rangle \mathrm{d} t-\frac{1}{8}\left(\frac{\sigma T}{T-t}\right)^{2}\left(\hat{H}-H_{t}\right)^{2}\left|\psi_{t}\right\rangle \mathrm{d} t+\frac{1}{2} \frac{\sigma T}{T-t}\left(\hat{H}-H_{t}\right)\left|\psi_{t}\right\rangle \mathrm{d} W_{t} .
$$

This model is of interest because the collapse of the wave function is achieved in finite time.

The stochastic equation (87) is identical to the finite-time collapse model introduced in [1], where a solution to (87) is obtained by considering an ansatz of the form

$$
\xi_{t}^{*}=\sigma t H+\beta_{t} .
$$

The noise term $\beta_{t}$ appearing here is a Brownian bridge [28, 36] that vanishes at $t=0$ and at $t=T$. The vanishing of the noise at $t=T$ guarantees the collapse of the wave function as $t$ approaches $T$. In particular, since the coupling $\sigma$ in (88) is constant, $\left\{\xi_{t}^{*}\right\}$ is a Markov process, as is shown in [12]. 
In the present framework, it follows immediately from (4) that the appropriate ansatz for solving (87) is given by

$$
\xi_{t}=\sigma T H \ln \left(\frac{T}{T-t}\right)+B_{t}
$$

where $\left\{B_{t}\right\}_{0 \leq t \leq T}$ is a standard Brownian motion. Remarkably, the two prescriptions (88) and (89) give rise to the same solution to the stochastic equation (87).

To see this we note that it follows from (16) that the energy process associated with (87) is given by

$$
H_{t}=\frac{\sum_{i} \pi_{i} E_{i} \exp \left(\sigma T E_{i} \int_{0}^{t} \frac{1}{T-s} \mathrm{~d} \xi_{s}-\frac{1}{2} \sigma^{2} E_{i}^{2} \frac{t T}{T-t}\right)}{\sum_{i} \pi_{i} \exp \left(\sigma T E_{i} \int_{0}^{t} \frac{1}{T-s} \mathrm{~d} \xi_{s}-\frac{1}{2} \sigma^{2} E_{i}^{2} \frac{t T}{T-t}\right)}
$$

In Section III we observed that under the probability measure $\mathbb{Q}$ the process $\left\{\xi_{t}\right\}$ is a Brownian motion. Therefore, in view of the expression appearing in the exponent of (90), we define a process $\left\{\xi_{t}^{*}\right\}$ according to the following scheme:

$$
\xi_{t}^{*}=(T-t) \int_{0}^{t} \frac{1}{T-s} \mathrm{~d} \xi_{s} .
$$

Substituting (89) into the right side of (91) we obtain

$$
(T-t) \int_{0}^{t} \frac{1}{T-s} \mathrm{~d} \xi_{s}=\sigma T H(T-t) \int_{0}^{t} \frac{1}{(T-s)^{2}} \mathrm{~d} s+(T-t) \int_{0}^{t} \frac{1}{T-s} \mathrm{~d} B_{s} .
$$

After a short calculation, we deduce that

$$
(T-t) \int_{0}^{t} \frac{1}{T-s} \mathrm{~d} \xi_{s}=\sigma t H+\beta_{t}
$$

where $\left\{\beta_{t}\right\}$ is defined by

$$
\beta_{t}=(T-t) \int_{0}^{t} \frac{1}{T-s} \mathrm{~d} B_{s}
$$

However, we recognise in (94) a standard integral representation of a Brownian bridge 28, 36]. It follows that $\left\{\xi_{t}^{*}\right\}$, as defined by (91), can be put into the form (88).

On the other hand, we also see that (91) is an integral representation of a Brownian bridge under $\mathbb{Q}$, since in this measure $\left\{\xi_{t}\right\}$ is a Brownian motion. Therefore, under $\mathbb{Q}$, the energy process (90) can be expressed in terms of a Brownian bridge $\left\{\xi_{t}^{*}\right\}$ in the form

$$
H_{t}=\frac{\sum_{i} \pi_{i} E_{i} \exp \left(\frac{T}{T-t}\left(\sigma E_{i} \xi_{t}^{*}-\frac{1}{2} \sigma^{2} E_{i}^{2} t\right)\right)}{\sum_{i} \pi_{i} \exp \left(\frac{T}{T-t}\left(\sigma T E_{i} \xi_{t}^{*}-\frac{1}{2} \sigma^{2} E_{i}^{2} t\right)\right)}
$$

This result agrees with the result obtained in [11] for the finite-time collapse model.

We note, incidentally, that the innovation process associated with $\left\{\xi_{t}\right\}$ in this example, given by

$$
W_{t}=\xi_{t}-\int_{0}^{t} \sigma_{s} H_{s} \mathrm{~d} s
$$


where $\sigma_{t}=\sigma T /(T-t)$, and the innovation process associated with $\left\{\xi_{t}^{*}\right\}$, given by

$$
W_{t}=\xi_{t}^{*}+\int_{0}^{t} \frac{1}{T-s}\left(\xi_{s}^{*}-\sigma T H_{s}\right) \mathrm{d} s,
$$

as obtained in [1], are identical if $\left\{\beta_{t}\right\}$ is defined as in (94). This follows on account of the relation

$$
\xi_{t}=\xi_{t}^{*}+\int_{0}^{t} \frac{1}{T-s} \xi_{s}^{*} \mathrm{~d} s
$$

which can be verified by writing the right side of (98) in differential form:

$$
\begin{aligned}
\mathrm{d} \xi_{t}^{*}+\frac{1}{T-t} \xi_{t}^{*} \mathrm{~d} t & =\sigma H \mathrm{~d} t+\mathrm{d} \beta_{t}+\frac{1}{T-t}\left(\sigma t H+\beta_{t}\right) \mathrm{d} t \\
& =\sigma H \mathrm{~d} t-\frac{1}{T-t} \beta_{t} \mathrm{~d} t+\mathrm{d} B_{t}+\sigma H \frac{t}{T-t} \mathrm{~d} t+\frac{1}{T-t} \beta_{t} \mathrm{~d} t \\
& =H \frac{\sigma T}{T-t} \mathrm{~d} t+\mathrm{d} B_{t}=\mathrm{d} \xi_{t} .
\end{aligned}
$$

Therefore, the solution obtained here in terms of $\left\{\xi_{t}\right\}$ is equivalent to the solution obtained in [1] using $\left\{\xi_{t}^{*}\right\}$. The results above show that models exhibiting state-vector reduction over a finite time horizon are both feasible and tractable, and that such models can be usefully formulated by use of a time-dependent coupling.

\section{Acknowledgments}

DCB acknowledges support from The Royal Society; ICC acknowledges support from UK Particle Physics and Astronomy Research Council; and JDCD and LPH acknowledge support from the UK Engineering and Physical Science Research Council (EPSRC). The authors thank S.L. Adler, C.M. Bender, I.R.C. Buckley, and M.H.A. Davis for useful discussions and correspondence, and an anonymous referee for helpful comments.

\section{APPENDIX A: PATH INTEGRAL APPROACH}

In this appendix we present a path integral method for calculating the conditional probability process $\left\{\pi_{i t}\right\}$ and the energy expectation process $\left\{H_{t}\right\}$. First we note that (7) can be written in the form:

$$
\mathbb{E}\left[H \mid\left\{\xi_{s}\right\}_{0 \leq s \leq t}\right]=\sum_{i} E_{i} \pi_{i t}
$$

where $\pi_{i t}=\mathbb{P}\left(H=E_{i} \mid\left\{\xi_{s}\right\}_{0 \leq s \leq t}\right)$. By use of the Bayes theorem, the conditional probability is given by

$$
\mathbb{P}\left(H=E_{i} \mid\left\{\xi_{s}\right\}_{0 \leq s \leq t}\right)=\frac{\pi_{i} \rho\left(\left\{\xi_{s}\right\}_{0 \leq s \leq t} \mid H=E_{i}\right)}{\sum_{i} \pi_{i} \rho\left(\left\{\xi_{s}\right\}_{0 \leq s \leq t} \mid H=E_{i}\right)}
$$


Here the expression

$$
\begin{aligned}
& \rho\left(\left\{\xi_{s}\right\}_{0 \leq s \leq t} \mid H=E_{i}\right) \\
& \quad=\sqrt{\frac{1}{\operatorname{det}(2 \pi \Sigma)}} \exp \left(-\frac{1}{2} \int_{0}^{t} \int_{0}^{t} \Sigma^{-1}(u, v)\left(\xi_{u}-E_{i} \int_{0}^{u} \sigma_{s} \mathrm{~d} s\right)\left(\xi_{v}-E_{i} \int_{0}^{v} \sigma_{s} \mathrm{~d} s\right) \mathrm{d} u \mathrm{~d} v\right)(\mathrm{A} 3
\end{aligned}
$$

is the density function of the trajectory $\left\{\xi_{s}\right\}_{0 \leq s \leq t}$ conditional on $H$ taking the value $E_{i}$. We write $\Sigma(u, v)$ for the covariance of the random variables $E_{i} \int_{0}^{u} \sigma_{s} \mathrm{~d} s+B_{u}$ and $E_{i} \int_{0}^{v} \sigma_{s} \mathrm{~d} s+B_{v}$. The form of the density function (A3) follows from that fact that, conditional on $H=E_{i}$, the random variables $\left\{\xi_{s}\right\}_{0 \leq s \leq t}$ are jointly normally distributed. A straightforward calculation making use of well-known properties of Brownian motion shows that

$$
\Sigma(u, v)=\min (u, v) .
$$

To compute the inverse $\Sigma^{-1}(u, v)$ of the covariance we substitute (A4) into the relation

$$
\int_{0}^{t} \Sigma^{-1}(u, s) \Sigma(s, v) \mathrm{d} s=\delta(u-v),
$$

and differentiate the resulting expression twice in the variable $v$ to obtain

$$
\Sigma^{-1}(u, v)=-\delta^{\prime \prime}(u-v),
$$

where $\delta^{\prime \prime}(t)$ denotes the second derivative of the Dirac delta function.

We note that the conditional expectation of any functional of the trajectory $\left\{\xi_{s}\right\}_{0 \leq s \leq t}$ can be determined by use of the density function (A3); the resulting expression corresponds to an infinite-dimensional Feynman integral. This is related to the fact that $\Sigma(u, v)$ gives the Feynman-Green function for a free particle [18, 20, 37].

To determine the form of the density function (A3), one might consider substituting (A6) into (A3) and then applying integration by parts, since $-\delta^{\prime \prime}(t)$ is a second-order differential operator (with the property that it has a positive "spike" at $t=0$ and a pair of negative "spikes" at $t=0^{+}$and $t=0^{-}$). However, the integral in the exponent of (A3) is ill-defined as it stands. Indeed, it is a straightforward exercise to verify that, depending on the order in which integration by parts is applied, one obtains different answers. To circumvent this difficulty we shall discretise the process $\left\{\xi_{t}\right\}$ first, derive the corresponding expression for the conditional density function (A2), and then take the continuum limit.

Our strategy for determining the conditional probability law for the trajectory $\left\{\xi_{s}\right\}_{0 \leq s \leq t}$ is thus as follows. Fixing $t$, we partition the range $[0, t]$ into $n$ equally-spaced intervals. In particular, we set $s_{k}=k \Delta(k=0,1, \ldots, n)$, where $\Delta=t / n$. We then calculate the covariance of the random variables $E_{i} \int_{0}^{s_{k}} \sigma_{u} \mathrm{~d} u+B_{s_{k}}$ and $E_{i} \int_{0}^{s_{l}} \sigma_{u} \mathrm{~d} u+B_{s_{l}}$, and obtain following expression for the covariance matrix (cf. [16]):

$$
\Sigma\left(s_{k}, s_{l}\right)=\left(\begin{array}{ccccccc}
s_{1} & s_{1} & s_{1} & s_{1} & \cdots & s_{1} & s_{1} \\
s_{1} & s_{2} & s_{2} & s_{2} & \cdots & s_{2} & s_{2} \\
s_{1} & s_{2} & s_{3} & s_{3} & \cdots & s_{3} & s_{3} \\
s_{1} & s_{2} & s_{3} & s_{4} & \cdots & s_{4} & s_{4} \\
\vdots & \vdots & \vdots & \vdots & \ddots & \vdots & \vdots \\
s_{1} & s_{2} & s_{3} & s_{4} & \cdots & s_{n-1} & s_{n-1} \\
s_{1} & s_{2} & s_{3} & s_{4} & \cdots & s_{n-1} & s_{n}
\end{array}\right) .
$$


The validity of this result should be evident from the continuous case (A4). The inverse of the covariance matrix is the following second-order difference operator:

$$
\left(\begin{array}{cccccc}
\frac{1}{s_{1}-s_{0}}+\frac{1}{s_{2}-s_{1}} & -\frac{1}{s_{2}-s_{1}} & 0 & 0 & \cdots & 0 \\
-\frac{1}{s_{2}-s_{1}} & \frac{1}{s_{2}-s_{1}}+\frac{1}{s_{3}-s_{2}} & -\frac{1}{s_{3}-s_{2}} & 0 & \ldots & 0 \\
0 & -\frac{1}{s_{3}-s_{2}} & \frac{1}{s_{3}-s_{2}}+\frac{1}{s_{4}-s_{3}} & -\frac{1}{s_{4}-s_{3}} & 0 & 0 \\
\vdots & \ddots & \ddots & \ddots & \ddots & \vdots \\
0 & \ldots & \cdots & -\frac{1}{s_{n-1}-s_{n-2}} & \frac{1}{s_{n-1}-s_{n-2}}+\frac{1}{s_{n}-s_{n-1}} & -\frac{1}{s_{n}-s_{n-1}} \\
0 & \cdots & \cdots & 0 & -\frac{1}{s_{n}-s_{n-1}} & \frac{1}{s_{n}-s_{n-1}}
\end{array}\right)
$$

Note that the last term on the diagonal is anomalous, and is different from the remaining terms on the diagonal. Because the partition of the interval $[0, t]$ is equally spaced, we have $s_{k}-s_{k-1}=\Delta$ for all $k=1,2, \ldots, n$. Therefore (A8) simplifies to

$$
\Sigma_{k l}^{-1}=\frac{1}{\Delta}\left(\begin{array}{ccccccc}
2 & -1 & 0 & \cdots & \cdots & \cdots & 0 \\
-1 & 2 & -1 & 0 & \cdots & \cdots & 0 \\
0 & -1 & 2 & -1 & 0 & \cdots & 0 \\
\vdots & \ddots & \ddots & \ddots & \ddots & \ddots & \vdots \\
0 & \cdots & 0 & -1 & 2 & -1 & 0 \\
0 & \cdots & \cdots & 0 & -1 & 2 & -1 \\
0 & \cdots & \cdots & \cdots & 0 & -1 & 1
\end{array}\right)
$$

For simplicity of notation let us write

$$
\alpha_{i}\left(s_{k}\right)=\xi_{s_{k}}-E_{i} \int_{0}^{s_{k}} \sigma_{u} \mathrm{~d} u .
$$

Then the discretised form of the conditional density function (A3) takes the form

$$
\begin{array}{r}
\rho\left(\left\{\xi_{s_{1}}, \xi_{s_{2}}, \ldots, \xi_{s_{n}}\right\} \mid H_{T}=E_{i}\right)=\left(\frac{\Delta}{2 \pi}\right)^{\frac{1}{2} n} \exp \left(-\frac{1}{2} \sum_{k=1}^{n} \sum_{l=1}^{n} \Sigma_{k l}^{-1} \alpha_{i}\left(s_{k}\right) \alpha_{i}\left(s_{l}\right)\right) \\
=\left(\frac{\Delta}{2 \pi}\right)^{\frac{1}{2} n} \exp \left(\frac{1}{\Delta} \sum_{k=1}^{n-1} \alpha_{i}\left(s_{k}\right)\left[\alpha_{i}\left(s_{k+1}\right)-\alpha_{i}\left(s_{k}\right)\right]-\frac{1}{2 \Delta} \alpha_{i}^{2}\left(s_{n}\right)\right) .
\end{array}
$$

Here we have used expression (A9) for the inverse covariance matrix. Let us examine the terms in the exponent. Substituting definition (A10) we find that

$$
\begin{aligned}
\alpha_{i}\left(s_{k}\right)[ & \left.\alpha_{i}\left(s_{k+1}\right)-\alpha_{i}\left(s_{k}\right)\right]=\xi_{s_{k}}\left(\xi_{s_{k+1}}-\xi_{s_{k}}\right)+E_{i}^{2} \int_{0}^{s_{k}} \sigma_{u} \mathrm{~d} u \int_{s_{k}}^{s_{k+1}} \sigma_{v} \mathrm{~d} v \\
& +E_{i}\left(\left(\xi_{s_{k+1}}-\xi_{s_{k}}\right) \int_{s_{k}}^{s_{k+1}} \sigma_{u} \mathrm{~d} u-\xi_{s_{k+1}} \int_{0}^{s_{k+1}} \sigma_{u} \mathrm{~d} u+\xi_{s_{k}} \int_{0}^{s_{k}} \sigma_{u} \mathrm{~d} u\right) .
\end{aligned}
$$

Turning to the conditional probability (A2) that we aim to determine, we observe by inspection of the right side of (A2) that all terms in the exponent that are independent of the eigenvalue $E_{i}$, such as the term $\xi_{s_{k}}\left(\xi_{s_{k+1}}-\xi_{s_{k}}\right)$ in the right side of (A12), cancel. This is because such terms appear in both the denominator and the numerator. Hence in 
what follows we omit such terms. Equality modulo omitted terms will be denoted by the symbol. Bearing this in mind, we see that the sum over $k$ of the right side of (A12) gives

$$
\begin{aligned}
\sum_{k=1}^{n-1} \alpha_{i}\left(s_{k}\right)[ & \left.\alpha_{i}\left(s_{k+1}\right)-\alpha_{i}\left(s_{k}\right)\right] \sim E_{i}^{2} \sum_{k=1}^{n-1} \int_{0}^{s_{k}} \sigma_{u} \mathrm{~d} u \int_{s_{k}}^{s_{k+1}} \sigma_{v} \mathrm{~d} v \\
& +E_{i}\left(\sum_{k=1}^{n-1} \sigma_{s_{k}}\left(\xi_{s_{k+1}}-\xi_{s_{k}}\right) \Delta-\xi_{s_{n}} \int_{0}^{s_{n}} \sigma_{u} \mathrm{~d} u+\xi_{s_{1}} \sigma_{s_{0}} \Delta\right)
\end{aligned}
$$

In deducing this result we have used the fact that for sufficiently large $n$, and hence sufficiently small $\Delta$, the following relation holds to a high degree of accuracy:

$$
\int_{s_{k}}^{s_{k+1}} \sigma_{u} \mathrm{~d} u=\sigma_{s_{k}} \Delta .
$$

Similarly, omitting the term that contains no $E_{i}$ we have

$$
\alpha_{i}^{2}\left(s_{n}\right) \sim-2 E_{i} \xi_{s_{n}} \int_{0}^{s_{n}} \sigma_{u} \mathrm{~d} u+E_{i}^{2}\left(\int_{0}^{s_{n}} \sigma_{u} \mathrm{~d} u\right)^{2} .
$$

Substituting (A13) and (A15) into (A11), we deduce that

$$
\rho\left(\left\{\xi_{s_{1}}, \xi_{s_{2}}, \ldots, \xi_{s_{n}}\right\} \mid H_{T}=E_{i}\right) \sim \exp \left(E_{i} \sum_{k=0}^{n-1} \sigma_{s_{k}}\left(\xi_{s_{k+1}}-\xi_{s_{k}}\right)-\frac{1}{2} E_{i}^{2} \sum_{k=0}^{n-1} \sigma_{s_{k}}^{2} \Delta\right) .
$$

Here we have made use of the fact that to a high degree of accuracy one has:

$$
\left(\int_{0}^{s_{n}} \sigma_{u} \mathrm{~d} u\right)^{2}=\sum_{k=0}^{n-1} \sigma_{s_{k}}^{2}(\Delta t)^{2}+2 \sum_{k \neq l}^{n-1} \sigma_{s_{k}} \sigma_{s_{l}} \Delta^{2}
$$

We have also used the fact that

$$
\sum_{k=1}^{n-1} \sum_{l=0}^{k-1} \sigma_{s_{k}} \sigma_{s_{l}}=\sum_{k \neq l}^{n-1} \sigma_{s_{k}} \sigma_{s_{l}}
$$

Note that the first sum in the exponent of (A16) begins from $k=0$, and not $k=1$ as in the right side of (A13). This is because the last term in the right side of (A13) can be written as $\sigma_{s_{0}}\left(\xi_{s_{1}}-\xi_{s_{0}}\right) \Delta$ (since $\left.\xi_{s_{0}}=0\right)$ and thus be absorbed in the sum.

We are now in the position to take the limit $n \rightarrow \infty$. In particular, the first term in the exponent of (A16) converges in this limit to an Ito integral of $\left\{\sigma_{t}\right\}$ with respect to the process $\left\{\xi_{t}\right\}$, since the discrete approximation is always taken to be the value of the integrand at $s_{k}$ in each interval $\left[s_{k}, s_{k+1}\right]$. The second sum, on the other hand, converges to the Riemann integral of the function $\left\{\sigma_{t}^{2}\right\}$. Writing $\left\{p_{i t}\right\}$ for the unnormalised density function given by the right side of (A16), we thus deduce, in the limit $n \rightarrow \infty$, that

$$
p_{i t}=\exp \left(E_{i} \int_{0}^{t} \sigma_{s} \mathrm{~d} \xi_{s}-\frac{1}{2} E_{i}^{2} \int_{0}^{t} \sigma_{s}^{2} \mathrm{~d} s\right) .
$$

As a consequence, for the conditional probability process $\left\{\pi_{i t}\right\}$ we obtain (15), as desired. 


\section{APPENDIX B: DECOMPOSITION OF PATH INTO INCREMENTS}

In this appendix we introduce another method for obtaining $\left\{\pi_{i t}\right\}$, based on the decomposition of the path of the quantum information process into its increments. The argument goes as follows. Recalling the dynamical equation (8) satisfied by the information process $\left\{\xi_{t}\right\}$, we note that $\left\{\sigma_{t}\right\}$ moderates the strength of the signal showing the true value of $H$. An equivalent set of information can be obtained by moderating the noise level by use of the reciprocal function $\left\{1 / \sigma_{t}\right\}$. Thus we consider a process $\left\{\zeta_{t}\right\}$ defined by

$$
\zeta_{t}=H t+\int_{0}^{t} \frac{1}{\sigma_{s}} \mathrm{~d} B_{s} .
$$

The relation between $\left\{\xi_{t}\right\}$ and $\left\{\zeta_{t}\right\}$ is given by

$$
\mathrm{d} \zeta_{t}=\frac{1}{\sigma_{t}} \mathrm{~d} \xi_{t}
$$

This alternative representation is motivated by Wonham [35], where the filtering equation for a signal associated with a fixed random variable is investigated.

The fact that the information implicit in $\left\{\xi_{s}\right\}_{0 \leq s \leq t}$ and $\left\{\zeta_{s}\right\}_{0 \leq s \leq t}$ is equivalent will be shown. We begin with the discretisation of the period $[0, t]$ into $n$ equally-spaced intervals of size $\Delta=t / n$, and set $s_{k}=k \Delta$ for $k=0,1, \ldots, n$. We define a process for the increments of $\left\{\zeta_{t}\right\}$ by setting $y_{s_{k}}=\zeta_{s_{k+1}}-\zeta_{s_{k}}$. It follows from (B1) that

$$
y_{s_{k}}=H \Delta+\int_{s_{k}}^{s_{k+1}} \sigma_{u}^{-1} \mathrm{~d} B_{u} .
$$

For each value of $n$, the random variables $y_{s_{k}}-H \Delta(k=0,1, \ldots, n-1)$ are independent and normally distributed, with mean zero and variance

$$
v_{s_{k}}=\int_{s_{k}}^{s_{k+1}} \sigma_{u}^{-2} \mathrm{~d} u .
$$

If we assume that $\Delta$ is small, then to a high degree of accuracy we have $v_{s_{k}}=\sigma_{s_{k}}^{-2} \Delta$. Next we note that

$$
\mathbb{P}\left(H=E_{i} \mid \zeta_{s_{0}}, \zeta_{s_{1}}, \cdots, \zeta_{s_{n}}\right)=\mathbb{P}\left(H=E_{i} \mid y_{s_{0}}, y_{s_{1}}, \cdots, y_{s_{n-1}}\right)
$$

This relation follows from the fact that conditioning with respect to $\left\{\zeta_{s_{k}}\right\}_{k=0,1, \ldots, n}$ is equivalent to conditioning with respect to the corresponding increments. Letting $\left\{\pi_{i t}^{(n)}\right\}$ denote the conditional probability defined by (B5), we conclude that

$$
\begin{aligned}
\pi_{i t}^{(n)}= & \frac{\pi_{i} \exp \left(-\frac{1}{2} \sum_{k=0}^{n-1} v_{s_{k}}^{-1}\left(y_{s_{k}}-E_{i} \Delta\right)^{2}\right)}{\sum_{i} \pi_{i} \exp \left(-\frac{1}{2} \sum_{k=0}^{n-1} v_{s_{k}}^{-1}\left(y_{s_{k}}-E_{i} \Delta\right)^{2}\right)} \\
= & \frac{\pi_{i} \exp \left(E_{i} \Delta \sum_{k=0}^{n-1} v_{s_{k}}^{-1} y_{s_{k}}-\frac{1}{2} E_{i}^{2} \sum_{k=0}^{n-1} v_{s_{k}}^{-1} \Delta^{2}\right)}{\sum_{i} \pi_{i} \exp \left(E_{i} \Delta \sum_{k=0}^{n-1} v_{s_{k}}^{-1} y_{s_{k}}-\frac{1}{2} E_{i}^{2} \sum_{k=0}^{n-1} v_{s_{k}}^{-1} \Delta^{2}\right)} .
\end{aligned}
$$


Substituting the right side of ( $(\underline{\mathrm{B} 4})$ into $(\underline{\mathrm{B} 6})$, and taking the limit as $n$ gets large, we have

$$
\pi_{i t}=\lim _{n \rightarrow \infty} \pi_{i t}^{(n)}=\frac{\pi_{i} \exp \left(E_{i} \int_{0}^{t} \sigma_{s}^{2} \mathrm{~d} \zeta_{s}-\frac{1}{2} E_{i}^{2} \int_{0}^{t} \sigma_{s}^{2} \mathrm{~d} s\right)}{\sum_{i} \pi_{i} \exp \left(E_{i} \int_{0}^{t} \sigma_{s}^{2} \mathrm{~d} \zeta_{s}-\frac{1}{2} E_{i}^{2} \int_{0}^{t} \sigma_{s}^{2} \mathrm{~d} s\right)}
$$

In taking the limit to obtain a stochastic integral we have followed a line of argument similar to that of Appendix A. This verifies that the information content of the trajectories $\left\{\xi_{s}\right\}_{0 \leq s \leq t}$ and $\left\{\zeta_{s}\right\}_{0 \leq s \leq t}$ is the same.

\section{References}

[1] S. L. Adler, "Environmental influence on the measurement process in stochastic reduction models" J. Phys. A35 841 (2002).

[2] S. L. Adler, "Weisskopf-Wigner decay theory for the energy-driven stochastic Schrödinger equation" Phys. Rev. D67 25007 (2003).

[3] S. L. Adler, Quantum Theory as an Emergent Phenomenon (Cambridge: Cambridge University Press, 2004).

[4] S. L. Adler, D. C. Brody, T. A. Brun, and L. P. Hughston, "Martingale models for quantum state reduction" J. Phys. A34 8795 (2001).

[5] S. L. Adler and T. A. Brun, "Generalized stochastic Schrödinger equations for state vector collapse" J. Phys. A34 4797 (2001).

[6] S. L. Adler and L. P. Horwitz, "Structure and properties of Hughston's stochastic extension of the Schrödinger equation" J. Math. Phys. 412485 (2000).

[7] A. Barchielli and V. P. Belavkin "Measurements continuous in time and a posteriori states in quantum mechanics" J. Phys. A24 1495 (1991).

[8] A. Bassi and G. C. Ghirardi "Dynamical reduction models" Phys. Rep. 379257 (2003).

[9] D. C. Brody and L. P. Hughston, "Stochastic reduction in nonlinear quantum mechanics" Proc. R. Soc. Lond. A458 1117 (2002).

[10] D. C. Brody and L. P. Hughston, "Efficient simulation of quantum state reduction" J. Math. Phys. 43, 5254 (2002).

[11] D. C. Brody and L. P. Hughston, "Finite-time stochastic reduction models" J. Math. Phys. 46, 082101 (2005).

[12] D. C. Brody and L. P. Hughston, "Quantum noise and stochastic reduction" J. Phys. A39 833 (2006).

[13] D. C. Brody, L. P. Hughston, and J. Syroka "Relaxation of quantum states under energy perturbations" Proc. R. Soc. Lond. A459 2297 (2003). 
[14] R. S. Bucy and P. D. Joseph, Filtering for stochastic processes with applications to guidance (New York: Interscience Publishers, 1968).

[15] H. J. Carmichael, ed., Special issue on stochastic quantum optics, Quantum and Semiclassical Optics 8, 49-314 (1996).

[16] H. J. Carmichael, Statistical Methods in Quantum Optics 1: Master Equations and Fokker-Planck Equations (Berlin: Springer Verlag, 1999).

[17] M. H. A. Davis and S. I. Marcus, "An introduction to nonlinear filtering" in Stochastic systems: The mathematics of filtering and identification and application, M. Hazewinkel and J. C. Willems, eds. (Dordrecht: D. Reidel, 1981).

[18] C. DeWitt-Morette, "Feynman path integrals" Commun. Math. Phys. 37, 63 (1974).

[19] L. Diosi, "Continuous quantum measurement and Ito formalism" Phys. Lett. A129 419 (1988).

[20] I. M. Gel'fand and A. M. Yagrom, "Integration in functional spaces and its applications in quantum physics" J. Math. Phys. 1, 48 (1960).

[21] G. C. Ghirardi, P. Pearle, and A. Rimini, "Markov processes in Hilbert space and continuous spontaneous localisation of systems of identical particles" Phys. Rev. A42, 78 (1990).

[22] N. Gisin, "Stochastic quantum dynamics and relativity" Helv. Phys. Acta 62, 363 (1989).

[23] N. Gisin and I. C. Percival, "The quantum-state diffusion model applied to open systems" J. Phys. A25, 5677 (1992).

[24] L. P. Hughston, "Geometry of stochastic state vector reduction" Proc. R. Soc. London A452, 953 (1996).

[25] C. J. Isham, Lectures on Quantum Theory (London: Imperial College Pres, 1995).

[26] T. Kailath, "The structure of Radon-Nikodym derivatives with respect to Wiener and related measure" Ann. Math. Statist. 42, 1054 (1971).

[27] G. Kallianpur and C. Striebel, "Estimation of stochastic systems: Arbitrary system process with additive white noise observation errors" Ann. Math. Statist. 39, 785 (1968).

[28] I. Karatzas and S. E. Shreve, Brownian Motion and Stochastic Calculus (Berlin: Springer, 1997).

[29] R. S. Liptser and A. N. Shiryaev, Statistics of Random Processes Vols. I and II, 2nd ed. (Berlin: Springer Verlag, 2000).

[30] G. Lüders "Über die Zustandsänderung durch den Messprozess" Ann. Physik 8, 322 (1951). 
[31] P. Pearle, 'Collapse Models" in Open Systems and Measurement in Relativistic Quantum Theory ed. H. P. Breuer and F. Petruccione (Heidelberg: Springer, 1999).

[32] P. Pearle, "Problems and aspects of energy-driven wave-function collapse models" Phys. Rev. A69, 042106 (2004).

[33] I. C. Percival, "Primary state diffusion" Proc. R. Soc. Lond. A447, 189 (1994).

[34] I. C. Percival, Quantum State Diffusion (Cambridge: Cambridge University Press, 1998).

[35] W. M. Wonham, "Some applications of stochastic differential equations to optimal nonlinear filtering" J. SIAM A2, 347 (1965).

[36] M. Yor, Some Aspects of Brownian Motion Part I \& II (Basel: Birkhäuser Verlag $1992 \& 1996)$.

[37] D. X. Xia, Measure and Integration Theory on Infinite-Dimensional Spaces (New York: Academic Press 1972). 